\title{
Absorption in X-ray spectra of high-redshift quasars
}

\section{Citation}

Elvis, Martin, Fabrizio Fiore, Belinda Wilkes, Jonathan McDowell, and Jill Bechtold. 1994. "Absorption in X-Ray Spectra of High-Redshift Quasars." The Astrophysical Journal 422 (February): 60. doi:10.1086/173703.

\section{Published Version}

doi:10.1086/173703

\section{Permanent link}

http://nrs.harvard.edu/urn-3:HUL.InstRepos:30212178

\section{Terms of Use}

This article was downloaded from Harvard University's DASH repository, and is made available under the terms and conditions applicable to Other Posted Material, as set forth at http:// nrs.harvard.edu/urn-3:HUL.InstRepos:dash.current.terms-of-use\#LAA

\section{Share Your Story}

The Harvard community has made this article openly available.

Please share how this access benefits you. Submit a story.

\section{Accessibility}


ABSORPTION IN X-RAY SPECTRA OF HIGH-REDSHIFT QUASARS

Martin Elvis, ${ }^{1}$ Fabrizio Fiore, Belinda Wilkes, and Jonathan McDowell

Harvard-Smithsonian Center for Astrophysics, 60 Garden Street, Cambridge, MA 02138

AND

JILL BECHTOLD

Steward Observatory, University of Arizona, Tucson, AZ 85721

Received 1993 April 16; accepted 1993 August 18

\begin{abstract}
We present evidence that $\mathrm{X}$-ray absorption is common in high-redshift quasars. We have studied six highredshift $(z \sim 3)$ quasars with the ROSAT PSPC of which four are in directions of low Galactic $N_{\mathrm{H}}$. Three out of these four show excess absorption, while only three in $\sim 50 z \lesssim 0.4$ quasars do, indicating that such absorption must be common, but not ubiquitous, at high redshifts, and that the absorbers must lie at $z>0.4$.

The six quasars were: S5 $0014+81$, Q0420-388, PKS 0438-436, S4 0636+680. PKS 2000-330, PKS 2126-158, which have redshifts between 2.85 and 3.78. PKS $0438-436$ and PKS 2126-158 show evidence for absorption above the local Galactic value at better than $99.999 \%$ confidence level. If the absorber is at the redshift of the quasar, then values of $N_{\mathrm{H}}=[0.86(+0.49,-0.28)] \times 10^{22}$ atoms $\mathrm{cm}^{-2}$ for PKS 0438-436, and $N_{\mathrm{H}}=[1.45(+1.20,-0.64)] \times 10^{22}$ atoms $\mathrm{cm}^{-2}$ for PKS $2126-158$, are implied, assuming solar abundances. The spectrum of S4 $0636+680$ also suggests the presence of a similarly large absorption column density at the $98 \%$ confidence level.

This absorption reverses the trend for the most luminous active galactic nuclei (AGN) to have the least $\mathrm{X}$-ray absorption, so a new mechanism is likely to be responsible. Intervening absorption due to damped Ly $\alpha$ systems is a plausible cause. We also suggest, as an intrinsic model, that intracluster material, e.g., a cooling flow, around the quasar could account for both the X-ray spectrum and other properties of these quasars. All the quasars are radio-loud and three are gigahertz peaked (two of the three showing absorption).

No excess absorption above the Galactic value is seen toward Q0420-388. This quasar has two damped Ly $\alpha$ systems at $z=3.08$. The limit on the X-ray column density implies a low ionization fraction, $N(\mathrm{H} \mathrm{I}) / N(\mathrm{H}) \gtrsim 4 \times 10^{-3}(3 \sigma)$, for solar abundances, for these systems, and can set a weak limit on the size of the absorber.

In the emitted frame these PSPC spectra cover the band $\sim 0.5-10 \mathrm{keV}$, which has been well observed for low-redshift quasars and AGN. Comparison of high and low-redshift spectra in this emitted band shows no change of mean spectral index greater than $\Delta \alpha_{E}>0.3(99 \%$ confidence) with either redshift or luminosity, for radio-loud quasars.
\end{abstract}

Subject headings: quasars: absorption lines - X-rays: galaxies

\section{INTRODUCTION}

The strong evolution shown by quasars with redshift must be fundamental in understanding what these objects are and how they form. By $z=3$ a typical quasar ${ }^{2}$ is $50-100$ times more luminous than a local one (Boyle, Fong, \& Shanks 1987; Boyle et al. 1993). The quasar continuum spans all wavelengths, yet quasars at redshifts above 1 have so far been studied in detail only in the optical and radio bands. In particular, the X-ray emission from quasars carries a significant fraction of their bolometric luminosity (e.g., Elvis et al. 1994). Since $\mathrm{X}$-rays are likely to come from the immediate neighborhood of the "central engine," the X-ray properties of high-redshift quasars will surely provide clues to understanding quasar evolution. X-ray spectra also provide a new means of studying the intervening systems along the line of sight to high-redshift quasars through photoelectric absorption appearing at low energies.

ROSAT is a good instrument for these studies because of its high sensitivity. Moreover, the rest energy range observed by

\footnotetext{
${ }^{1}$ Electronic mail: I: elvis@cfa.harvard.edu.

2 That is, one at $L^{*}$, the luminosity at which the luminosity function changes slope.
}

$\operatorname{ROSAT}$ at $z=3$ is $\sim 0.5-10 \mathrm{keV}$, which is similar to the range in which most of the low-redshift quasars have been observed with the EXOSAT and Ginga satellites. This makes it possible to search for evolutionary changes in the same emitted energy band by comparing ROSAT spectra of high-redshift quasars with already measured spectra of objects at low redshift.

We have initiated a program to study high- $z$ quasars with the ROSAT PSPC. Wilkes et al. (1992) reported a first detection of excess absorption toward a high-z quasar. We now have observations of six quasars with redshifts ranging from 2.85 to 3.78 , and we find two more cases of likely strong absorption in their X-ray spectra.

In this paper we present spectra for the four quasars at $z \gtrsim 3$ for which we have more than 350 counts, and simple $\mathrm{X}$-ray colors for two $z>3$ quasars which have $\sim 60$ counts. A separate paper will discuss the X-ray fluxes and overall spectral energy distributions of all the quasars for which we have detections (Bechtold et al. 1994). Observational feasibility constrained our selection of objects to those with either known high X-ray fluxes or bright apparent optical magnitudes. Combined with the requirement of high redshift, this biases our quasar sample toward the most luminous objects in the universe. 
We assume a Friedmann cosmology with $H_{0}=50 \mathrm{~km} \mathrm{~s}^{-1}$ $\mathrm{Mpc}^{-1}$ and $\Omega_{0}=0$.

\section{OBSERVATIONS}

ROSAT (Trümper 1983) observed the high-redshift quasars reported here with the Position Sensitive Proportional Counter (PSPC, Pfefferman et al. 1987) between 1991 February and 1992 September. Details are given in Table 1. Each of the quasars was detected as an X-ray source, and the PSPC derived position for the quasars lies within $12^{\prime \prime}$ of the accurate optical positions of Schneider et al. (1992) with exception of the 1991 February observation of PKS 0438-436. The PSPC position in the Standard Analysis Software System (SASS) processing for the X-ray source identified with PKS 0438-436 in this observation is 2.9 from the quasar optical position. However, we are confident of the identification because a second quasar in the field, PKS 0439-433, is also offset in the same direction by 2.6 . The difference in offsets of $18^{\prime \prime}$ is similar to the PSPC beam size. PKS $0439-433$ is well off-axis and close to a support structure which may induce an additional slight offset. Aspect errors of this size have been seen in a few other ROSAT pointings with the current version of the SASS (ROSAT Newsletter no. 10, 1992). Also, the second observation in 1992 September gives a position for PKS 0438-436 within 6 " of the optical position.

For six of the observations the standard "wobble" mode was on (Table 1). In this mode the detector is moved to and fro along a $3^{\prime}$ path with a $400 \mathrm{~s}$ period, to smooth out detector structure. In the other two observations, the wobble was turned off. The source counts reported in Table 1 are net counts within a circle of radius $2^{\prime}$ around the X-ray centroid of the source, enclosing $95 \%$ of the counts in a point source (George \& Turner 1992). For the wobbled observations and for the observation of Q0420-388, the background spectra were taken from an annulus of inner and outer radii 2.5 and $5^{\prime}$. Background subtraction for the unwobbled observation of PKS $0438-436$ is described below. The source counts do not increase significantly considering broader source and background regions both in the $0.1-2.5 \mathrm{keV}$ and the $0.1-0.3 \mathrm{keV}$ energy bands, suggesting that any "electronic ghost images" that form at low energies, below $\sim 0.15 \mathrm{keV}$ (George \& Turner 1992), are unimportant in these observations. The 34 energy channels from the MPE SASS pipeline processing were used in this analysis.

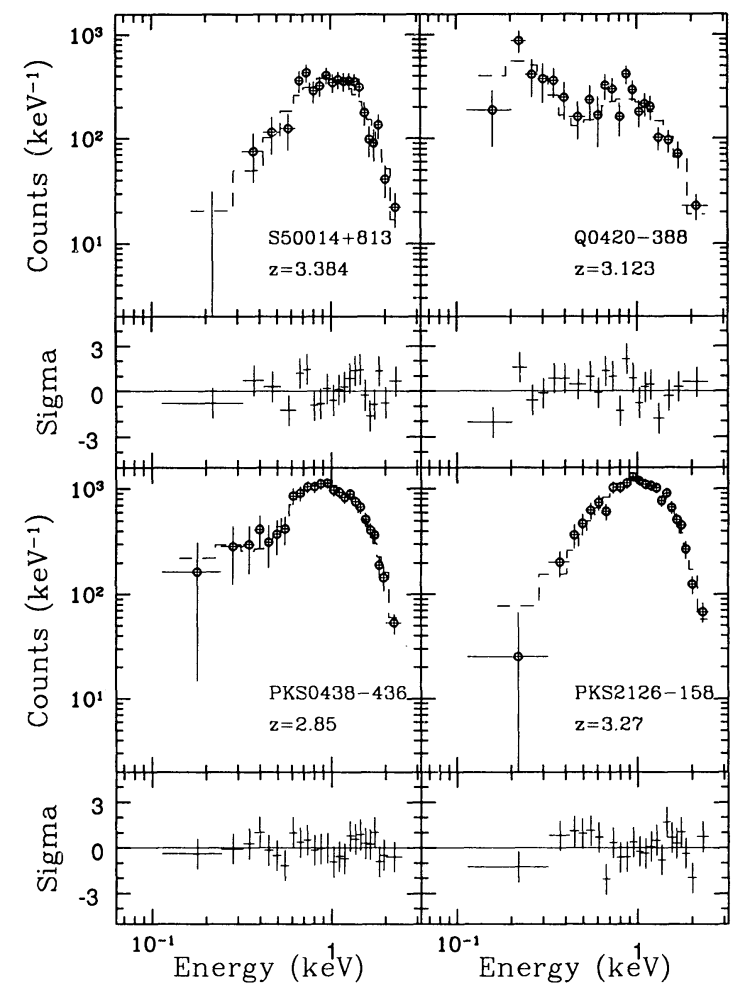

FIG. 1.-ROSAT PSPC pulse height spectra for the four high $z$ quasars. The upper panel in each case shows the pulse height spectrum and best-fit power-law plus absorption model, and the lower panel shows the residuals from these fits. The fits shown are (a) S5 $0014+813$ and (b) Q0420-388: $N_{\mathrm{H}}$ fixed at the Galactic value; (c) PKS 0438-436 (co-added spectrum), and (d) PKS 2126-158 (co-added spectrum): $N_{\mathrm{H}}$ at the best-fit value in the quasar frame.

The pulse height (PHA) spectra are shown in Figure 1 for the four quasars with more than 350 counts. Three of these show a marked absence of low-energy counts. Such low-energy cutoffs are a characteristic signature of photoelectric absorption. To quantify this impression fits of simple power laws with the flux reduced at low energies by photoelectric absorption due to cold material at cosmic abundances (cross sections from Morrison \& McCammon 1983) were made (Table 2, fit 1). In Table 2 we give the values of the fitted parameters, the normalization,

TABLE 1

ROSAT PSPC OBSERVATIONS OF High-REDSHIFT QUASARS

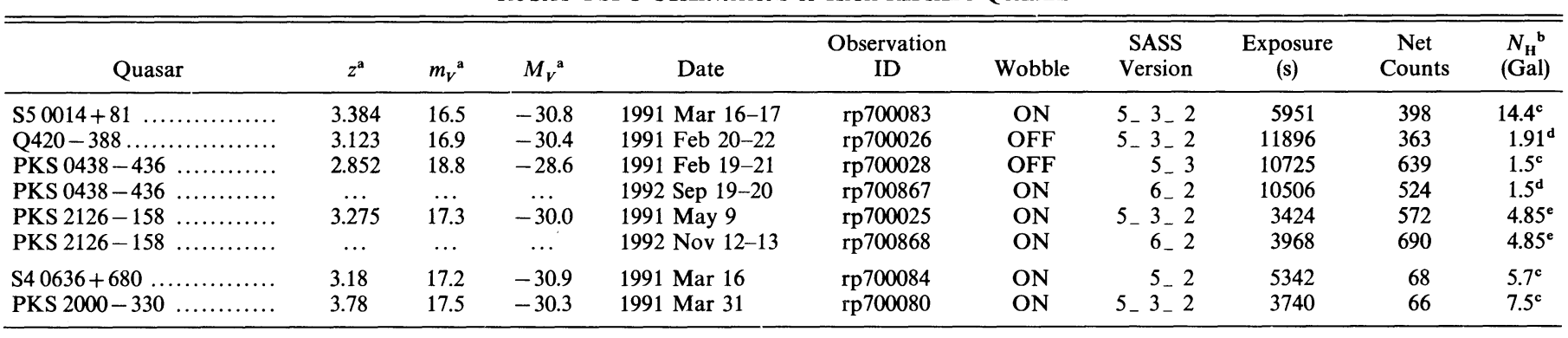

${ }^{a}$ Véron-Cetty \& Véron 1991.

b In units of $10^{20}$ atoms $\mathrm{cm}^{-2}$.

c Stark et al. 1989.

d Heiles \& Cleary 1979.

e Elvis, Lockman, \& Wilkes 1989. 
TABLE 2

PSPC X-RAY SPECTRAL Fits FOR High-REDShift QUASARS

\begin{tabular}{|c|c|c|c|c|c|c|}
\hline Quasar & Fit & Normalization $^{\mathrm{a}}$ & $\alpha_{E}$ & $N_{\mathbf{H}}^{\mathrm{b}}$ & $\chi_{v}^{2}(\mathrm{dof})$ & $\mathrm{Fe}-\mathrm{K} \mathrm{EW}$ \\
\hline \multirow{3}{*}{ 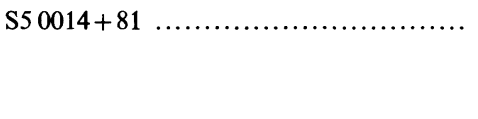 } & 1 & $5.5_{-1.1}^{+2.6}$ & $1.07_{-0.73}^{+1.2}$ & $19_{-8}^{+24}$ & $1.06(18)$ & $<1.1$ \\
\hline & 2 & $4.7 \pm 0.27$ & $0.82 \pm 0.19$ & 14.4 Fixed & $1.02(19)$ & \\
\hline & 3 & $5.0_{-0.6}^{+2.0}$ & $0.92 \pm 0.67$ & $64_{-64}^{+500}$ & $1.07(18)$ & \\
\hline \multirow{3}{*}{ Q0420-388 $\ldots \ldots \ldots \ldots \ldots \ldots \ldots \ldots \ldots \ldots \ldots \ldots \ldots \ldots$} & 1 & $1.10 \pm 0.14$ & $1.27 \pm 0.50$ & $3.6_{-1.4}^{+1.7}$ & $1.15(18)$ & $<1.4$ \\
\hline & 2 & $1.01 \pm 0.07$ & $0.73 \pm 0.10$ & 1.91 Fixed & $1.27(19)$ & \\
\hline & 3 & $1.08 \pm 0.13$ & $1.24 \pm 0.48$ & $34 \pm 33$ & $1.17(18)$ & \\
\hline PKS $0438-436(1991 \mathrm{Feb})$. & 1 & $3.6_{-0.4}^{+0.8}$ & $0.83_{-0.35}^{+0.61}$ & $8.6_{-3.2}^{+10.6}$ & $1.10(21)$ & \\
\hline PKS $0438-436(1992$ Sep) $\ldots \ldots \ldots \ldots \ldots$ & 1 & $2.6_{-0.3}^{+0.4}$ & $0.67_{-0.33}^{+0.38}$ & $6.5_{-2.2}^{+4.8}$ & $1.22(21)$ & \\
\hline \multirow{3}{*}{ PKS $0438-436$ (tot.) $\ldots \ldots \ldots \ldots \ldots \ldots \ldots$} & 1 & $3.11_{-0.28}^{+0.38}$ & $0.70_{-0.22}^{+0.27}$ & $6.9_{-1.8}^{+3.5}$ & $0.47(22)$ & \\
\hline & 2 & $2.35 \pm 0.08$ & $-0.155 \pm 0.06$ & 1.5 Fixed & $2.41(23)$ & \\
\hline & 3 & $2.91_{-0.19}^{+0.22}$ & $0.61 \pm 0.22$ & $86_{-28}^{+49}$ & $0.47(22)$ & $<0.78$ \\
\hline PKS 2126-158(1991 May) ............. & 1 & $10.4_{-1.6}^{+3.9}$ & $0.52 \pm 0.44$ & $11_{-4}^{+11}$ & $1.37(20)$ & \\
\hline PKS 2126-158(1992 Nov) $\ldots \ldots \ldots \ldots \ldots$ & 1 & $12.4_{-2.2}^{+5.7}$ & $0.86_{-0.43}^{+0.72}$ & $14.8_{-5.7}^{+13.2}$ & $0.86(20)$ & \\
\hline \multirow[t]{3}{*}{ PKS $2126-158$ (tot.) $\ldots \ldots \ldots \ldots \ldots \ldots \ldots$} & 1 & $11.6_{-1.4}^{+2.7}$ & $0.70_{-0.29}^{+0.41}$ & $12.9_{-3.8}^{+7.2}$ & $1.03(20)$ & \\
\hline & 2 & $8.6 \pm 0.3$ & $-0.03 \pm 0.07$ & 4.85 Fixed & $2.36(21)$ & \\
\hline & 3 & $10.3 \pm 1.2$ & $0.52_{-0.21}^{+0.25}$ & $145_{-63}^{+120}$ & $1.09(20)$ & $0.5 \pm 0.5$ \\
\hline$S 40636+680 \ldots \ldots \ldots \ldots \ldots \ldots \ldots \ldots \ldots \ldots \ldots \ldots \ldots \ldots \ldots$ & 4 & $1.2 \pm 0.3$ & 0.71 Fixed & $590_{-340}^{+600 d}$ & $0.82(6)$ & \\
\hline PKS $2000-330 \ldots \ldots \ldots \ldots \ldots \ldots \ldots \ldots$ & 4 & $0.9 \pm 0.2$ & 0.71 Fixed & $0+220^{d}$ & $1.53(7)$ & \\
\hline
\end{tabular}

a In units of $10^{-4} \mathrm{~cm}^{-2} \mathrm{~s}^{-1} \mathrm{keV}^{-1} @ 1 \mathrm{keV}$.

b In units of $10^{20} \mathrm{~cm}^{-2}$.

c $90 \%$ upper limit (in units of $\mathrm{keV}$ ) for two interesting parameters at $6.4 \mathrm{keV}$ in the emitted frame.

d Absorber in the quasar frame (plus Galactic absorption).

Fit: $1-z=0, N_{\mathrm{H}}$, free; $2-z=0, N_{\mathrm{H}}$, fixed at Galactic $\mathrm{H}$ I value; 3 -absorber in the quasar frame, plus absorption fixed at Galactic $\mathrm{H}$ I value; 4 -spectral index fixed at the mean value from the fits to the previous four quasars, absorber in the quasar frame, plus absorption fixed at the Galactic $\mathrm{H}$ i value.

the energy spectral index $\alpha_{E}$, and the column density $N_{\mathbf{H}}$ (the errors represent the $68 \%$ confidence intervals for two interesting parameters when both $\alpha_{E}$ and $N_{H}$ are free to vary, and for a single interesting parameter when $N_{H}$ is fixed). We also list in Table 2 the reduced $\chi^{2}$ for the fit and the number of degrees of freedom (dof). The 1993 January version of the PSPC response matrix (DRM_36_1) was used in this analysis. The fitted parameters do not differ significantly from those obtained with the 1992 March matrix (DRM_9), suggesting that calibration systematic uncertainties are small and neglible in comparison to the statistical uncertainties. In the analysis we included, however, a systematic error of $1 \%$, as suggested by Hasinger \& Snowden (1992). The fits were performed over the $0.1-2.45 \mathrm{keV}$ energy range (channels $3-34)$.

In all cases the $\chi^{2}$ values are consistent with the simple power law plus absorption model (fit 1), which is unsurprising given the limited number of counts in the spectra. For two of the quasars, though, the allowed values of $N_{\mathrm{H}}$ are larger than the Galactic value. Fits with $N_{H}$ fixed at the Galactic value (fit 2) do not give acceptable fits for these quasars. Figure 2 shows $68 \%, 90 \%$, and $99 \%$ confidence contours in $\left(\log N_{\mathrm{H}}, \alpha\right)$ space (solid lines). These do not overlap the Galactic value of $N_{\mathrm{H}}$ for PKS $0438-436$ or PKS 2126-158. Fits with an additional absorber at the redshift of the quasars (fit 3) suggest large column densities (Fig. 1 fits and residuals, Fig. 2-thicker lines).

The X-ray absorption cutoff, although conventionally reported as a hydrogen column density, in fact measures the total column density toward the object, not just the neutral hydrogen column. Around a kilovolt, where the cutoffs in the X-ray spectra of PKS 2126-158 and PKS 0438-436 are seen (in the quasar frame), the X-ray absorption cross sections for metals (primarily the K-edges of oxygen, and to a lesser extent to $\mathrm{C}, \mathrm{Ne}, \mathrm{Mg}, \mathrm{Si}, \mathrm{S}$, and $\mathrm{Fe}$ ) are 5-10 times larger than the $\mathrm{H}+\mathrm{He}$ cross sections (Morrison \& McCammon 1983). The metals can be in small dust grains and still produce $\mathrm{X}$-ray

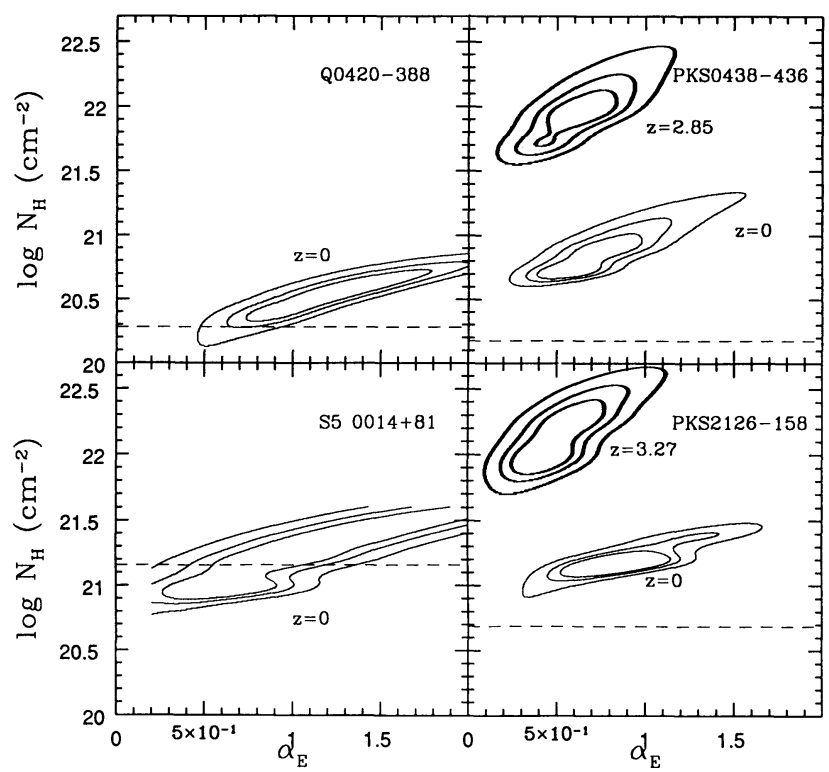

FIG. 2.-Contours of allowed values of $\alpha_{E}$ and $N_{\mathrm{H}}$ for the four high $z$ quasars. The thin solid contours are for $z=0$ absorption, which can be compared with the Galactic $\mathrm{H}_{\mathrm{I}}$ values (horizontal dashed lines). The thick contours are for absorption at the quasar redshift. Contours are $68 \%, 90 \%$, and $99 \%$ confidence for two interesting parameters. 


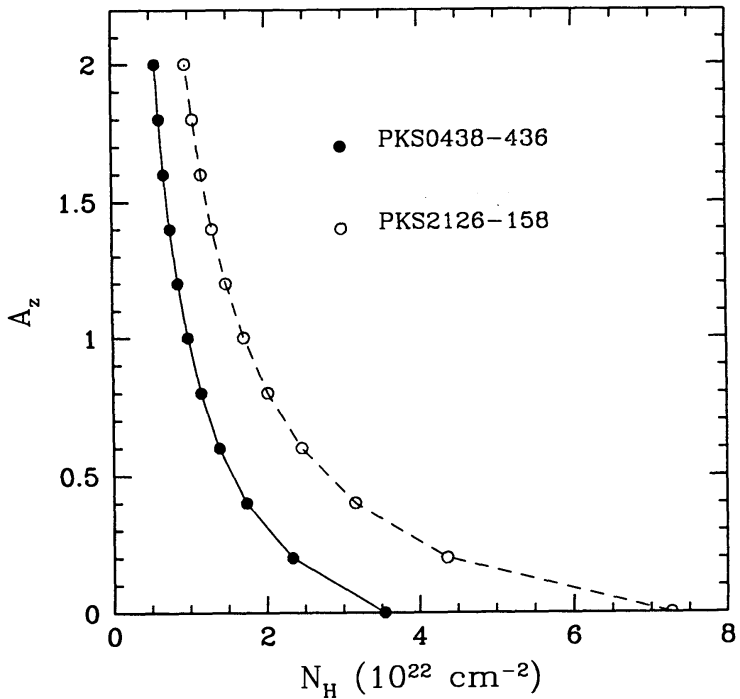

FIG. 3.-The dependence of the ratio $A_{z}$, the metal abundances relative to the solar value, on $N_{\mathrm{H}}$ at the quasar redshift for PKS 0438-436 (filled circles) and PKS 2126-158 (open circles).

absorption (until the grains grow big enough to shield their inner parts), with only minor modifications to their cross sections (Fireman 1974). Only fully ionized material has no effect. Since metal abundances at high redshift could well be $1 / 10$ solar or less (Meyer \& Roth 1990; Pettini, Boksenberg, \& Hunstead 1990; Turnshek et al. 1989), we have also made power-law spectral fits with an absorber of varying abundance at the quasar redshift. For a sake of simplicity the relative abundance among different metals have been fixed at the Morrison \& McCammon (1983) values. We therefore increased the number of degrees of freedom only by one, the ratio $A_{z}$ of the metal abundances ( $\mathrm{C}, \mathrm{N}, \mathrm{O}, \mathrm{Ne}, \mathrm{Mg}, \mathrm{Si}, \mathrm{S}, \mathrm{Ar}, \mathrm{Ca}$, and $\mathrm{Fe}$ ) at the quasar redshift to the solar ones. The fits to the co-added spectra of PKS 0438-436 and PKS 2126-158 (see $\$ 2.3$ and 2.4 ) give the same $\chi^{2}$, and spectral indices (to within \pm 0.05 ) for a range of $R$ from 0 to 2 . The PSPC cannot distinguish the shape of solar and zero metal abundance absorption in these high- $z$ quasars. As would be expected $A_{z}$ is strongly anticorrelated with $N_{\mathrm{H}}$, as is shown in Figure 3, where we plot the best-fit values of the two parameters for both quasars. When assuming only the $\mathrm{H}+\mathrm{He}$ cross sections appropriate to primordial material $\left(A_{z}=0\right)$, the $N_{\mathrm{H}}$ required to fit the data adequately is about 4 times that obtained when $A_{z}=1$ [while $\left.N_{\mathrm{H}}\left(A_{z}=0.1\right) \sim 3 N_{\mathrm{H}}\left(A_{z}=1\right)\right]$. At higher $A_{z}$ the $N_{\mathrm{H}}$ values decrease inversely with $A_{z}$.

The PSPC does not constrain the redshift of the absorbing material. The derived column density increases as $z_{\text {absorber }}$ is increased, since the absorbed photons gain energy as $(1+z)$. Figure 4 shows the constraints on the $N_{\mathrm{H}}$ of the absorbing material as a function of its assumed redshift for the two quasars with strong $N_{\mathrm{H}}$.

A power law with photoelectric absorption is not the only possible model that can give low-energy cutoffs. We also fitted blackbody models to the PSPC spectra. These fits gave best-fit temperatures of $0.9-1.6 \mathrm{keV}$, so that the low-energy cutoff is modeled as the Rayleigh-Jeans tail. In all four cases, however, the $\chi^{2}$ values were worse than for the power laws and were rejectable at the $95 \%$ level or higher. Given the limited energy resolution of proportional counters, strong Fe-L line emission from an optically thin plasma at around $1 \mathrm{keV}$ (Raymond \& Smith 1977) can simulate a power law with a low-energy cutoff. However, at $z=3$, this emission is redshifted to $\sim \frac{1}{4} \mathrm{keV}$ where it would produce a soft excess rather than a deficit. The $\sim 4$ $\mathrm{keV}$ region that is redshifted into the $1 \mathrm{keV}$ band is relatively free of strong emission-line features. Fits to Raymond plasmas gave acceptable $\chi^{2}$ values (for $50 \%$ solar abundances, with redshifts fixed at the quasar value), but only for temperatures above the range that can be well determined by $R O S A T$ at this signal-to-noise ( $k T=4-13 \mathrm{keV})$, as well as excess column densities. Such high temperatures have little line emission so that in the PSPC range they are essentially power laws. Since power-law fits are better constrained, we did not consider optically thin thermal models further. It should be noted that no
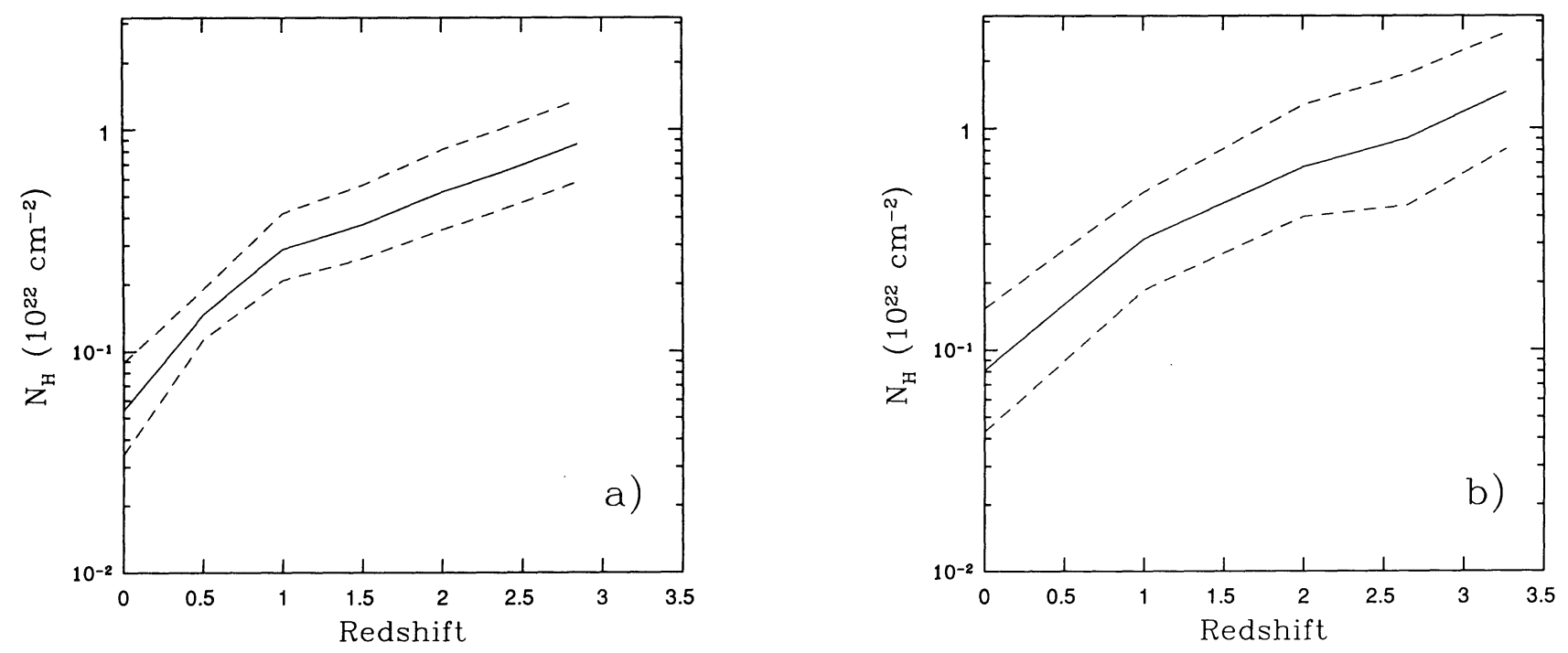

FIG. 4.- The dependence of the deduced equivalent hydrogen density on the redshift assumed for the absorber along the line of sight to $(a)$ PKS $0438-436$ and $(b)$ PKS 126-158 (solid line). The dashed lines show the $68 \%$ confidence region for this absorption. 
simple model need be appropriate. Complex X-ray spectra in active galactic nuclei (AGN) are becoming required as signalto-noise and resolution improve (e.g., Fiore et al. 1993; Marshall et al. 1993). While better measurements are needed, we shall adopt the straightfoward absorbed power-law model in the remainder of this paper.

The observed energy of the iron $\mathrm{K}$ fluorescence line $(6.4 \mathrm{keV}$ rest frame) is with the ROSAT band for $z>2$. ROSAT observations of high-redshift quasars can therefore constrain the amount of K-fluorescence emission in these quasars, albeit weakly. We fitted a power law plus absorption model (model 3), adding a narrow Gaussian line at the fixed, redshifted energy of the $6.4 \mathrm{keV}$ Fe-K fluorescence line to the PSPC spectra. These limits on rest equivalent width are also given in Table 2 and lie in the range $0.7-1.5 \mathrm{keV}$ ( $90 \%$ confidence).

Table 3 gives fluxes and luminosities derived from the single power-law fits for each quasar. Systematic errors for the wobbled observations are $\sim 4 \%$ (E. Schlegel, private communication). Uncertainties in the luminosities quoted are dominated by the choice of cosmological parameters. The luminosities in Table 3 are reduced by factors of about 4 for $\Omega_{0}=1$, depending on each object's redshift. For reference $3 \mathrm{C}$ 273 has a luminosity of $1 \times 10^{46} \mathrm{ergs} \mathrm{s}^{-1}(2-10 \mathrm{keV}$, Williams et al. 1992), $1 \%-10 \%$ of the luminosity of these quasars.

Below we give some details on each of the quasars.

\section{1. $S 50014+813, z=3.38$}

This radio-loud quasar is bright in $\mathrm{X}$-rays, with an observed $0.1-2.5 \mathrm{keV}$ flux of about $2 \times 10^{-12} \mathrm{ergs} \mathrm{cm}^{-2} \mathrm{~s}^{-1}$. It is the only $z>3$ quasar to have a previously reported X-ray spectrum (Lawson et al. 1992), obtained with EXOSAT.

The best-fit value of the column density $N_{\mathrm{H}}$ is consistent with the rather large Galactic value of $1.4 \times 10^{21} \mathrm{~cm}^{-2}$, as the lack of low-energy counts in Figure $1 a$ suggests. If the $N_{\mathrm{H}}$ is fixed to the Galactic value, the spectral index obtained is $\alpha_{E}=$ $0.82 \pm 0.19$ ( $1 \sigma$ error for one interesting parameter).

This quasar was observed with EXOSAT in 1984 November. The spectral index reported by Lawson et al. (1992) is $0.9 \pm 0.4$ (1 $\sigma$ error for one interesting parameter), thus suggesting that the 4-40 keV (rest frame) spectrum does not present large departures from the power-law slope found by ROSAT. S5 0014+813 was not observed with the Einstein

TABLE 3

FluXes AND LUMINOSITIES OF High-REDSHIFT QUASARS

\begin{tabular}{|c|c|c|c|}
\hline Quasar & Fit & $f_{X}^{\mathrm{a}}$ & $L_{X}^{\mathrm{b}}$ \\
\hline S5 $0014+81 \ldots$ & 2 & $22_{-3}^{+4}$ & $44_{-3.4}^{+3.9}$ \\
\hline Q0420-388 ... & 2 & $4.8^{\mathrm{c}}$ & $7.7^{\mathrm{c}}$ \\
\hline PKS $0438-436(1991 \mathrm{Feb})$ & 3 & $14^{\mathrm{c}}$ & $18^{\mathrm{c}}$ \\
\hline PKS $0438-436(1992$ Sep $) ..$ & 3 & $10_{-1.4}^{+2.7}$ & $14_{-2.1}^{+2.5}$ \\
\hline PKS $0438-436$ (total) ..... & 3 & $12^{\mathrm{c}}$ & $16^{\mathrm{c}}$ \\
\hline PKS 2126- 158 (total) & 3 & $42_{-6.6}^{+9.7}$ & $88_{-12}^{+15}$ \\
\hline$S 40636+680$ & 4 & $5.2_{-1.4}^{+1.4}$ & $9.2_{-2.4}^{+2.4}$ \\
\hline PKS $2000-330 \ldots \ldots \ldots \ldots \ldots \ldots \ldots \ldots$ & 4 & $3.9_{-0.9}^{+0.9}$ & $11.7_{-2.7}^{+2.7}$ \\
\hline
\end{tabular}

a $0.1-2.5 \mathrm{keV}$ unabsorbed flux in units of $10^{-13} \mathrm{ergs} \mathrm{cm}^{-2} \mathrm{~s}^{-1} \mathrm{keV}^{-1}$

b $2-10 \mathrm{keV}$ luminosity in units of $10^{46} \mathrm{ergs} \mathrm{s}^{-1}\left(H_{\mathrm{o}}=50 \mathrm{~km} \mathrm{~s}^{-1} \mathrm{Mpc}^{-1}\right.$, $\Omega_{0}=0$ ), assuming isotropic emission. The luminosities change by factors of about 4 for $\Omega_{0}=1$, depending on each object's redshift.

c Wobble off. Systematic uncertainties can be as large as a factor 2, mostly in the direction of the observed fluxes being underestimates.
IPC, since it had not been discovered when Einstein was functioning.

$$
\text { 2.2. } Q 0420-388, z=3.12
$$

This is the only optically selected (Osmer \& Smith 1977) quasar in our sample with sufficient counts to obtain a good spectrum (shown in Fig. 1b). It is not, however, radio-quiet. In fact, Q0420-388 is quite a strong radio source (Condon et al. 1981). Radio-loudness is normally evaluated between the optical $B$ band and $5 \mathrm{GHz}, R_{L}=\log \left[f_{(5 \mathrm{GHz})} / f_{B}\right]$, with radioloud objects having $R_{L}>1$. Q0420-388 has $R_{L}=2.24$ (using Condon et al. 1981 for the $1.4 \mathrm{GHz}$ radio flux and Hyland \& Allen 1982 for the $H$ magnitude, which lie closest to the emitted fiducial frequencies), and so is radio-loud. Q0420-388 is also a candidate gigahertz peaked spectrum source (GPS, O'Dea 1990), with a cutoff at $\sim 1.5 \mathrm{GHz}$ (observed frame, Condon et al. 1981).

There were problems in two of the eight observing intervals ("OBI") of this observation, due to mispointing of the satellite. For the present analysis we used only the first four and the last two observing intervals, giving a total integration time of $11,896 \mathrm{~s}$. Although the wobble was off for this observation, the standard source and background regions were used since the problems encountered for PKS 0438-436 (see below) were not present. The results are stable if the background region is moved by small amounts.

The large number of low-energy counts suggests little absorption and the best-fit value of $N_{\mathrm{H}}$ is consistent with the low Galactic value of $1.9 \times 10^{20}$ atoms $\mathrm{cm}^{-2}$ (Fig. 2). The spectral index obtained by fixing $N_{\mathbf{H}}$ to the Galactic value is $\alpha_{E}=0.73 \pm 0.10$, consistent with the mean value found in radio-loud low-redshift quasars in the $1-10 \mathrm{keV}$ rest frame band (Williams et al. 1992; Lawson et al. 1992), and in the 0.2-3.4 keV band (Wilkes \& Elvis 1987; Shastri et al. 1993).

The PSPC count rate of 0.031 counts $\mathrm{s}^{-1}$ is about double the 1979 Einstein IPC count rate of $0.016 \mathrm{ct} \mathrm{s}^{-1}$ (Harris et al. 1990). Given the relative sensitivity of the telescopes this is consistent with no flux variations between the two observations (ROSAT Mission Description 1991), assuming the bestfit PSPC spectrum.

\section{3. $P K S 0438-436, z=2.85$}

We now have double the exposure time on this quasar compared with that reported by Wilkes et al. (1992), thanks to a second observation 18 months after the first (Table 1). We first repeated the analysis of the 1991 February observation following Wilkes et al. (1992) but using a larger region to collect the lowest energy counts. (The "electronic ghost images" problem was only widely known after that paper was completed.) This results in $7 \%$ more counts and a better fit at low energies. The effect of the wobble being off in this observation is that an estimate of the background from our standard annulus around the source gives negative counts in the first six channels. This is because the background just around source is lower than average (probably due to shadowing by the wires supporting the window), while our standard background annulus captures a higher, and inappropriate, background. The best background subtraction (the one which minimizes the problem in the first channels) is obtained by taking the background along the shadow of the wires in a rectangular region to the south of the source. Adding these low-energy counts does not remove the absorption reported by Wilkes et al. (1992). The power-law slope is better constrained on the steep side in our new fit (Table 2). During the second, 1992 September, observation the 
wobble was on and the background subtraction was performed as described at the beginning of this section.

The 1991 February and 1992 September pulse height spectra were very similar, as were the best-fit parameters (Table 2) with exception of the normalization, which is $40 \%$ lower in the second observation (a $1.8 \sigma$ difference). Due to the lack of spacecraft wobble, the flux measured during the earlier observation is a lower limit to the real source flux, as the fraction of the observation for which the source may have been occulted by the support wires is uncertain. The real source flux could be as high as twice the value reported in Table 3 . It is therefore possible, but not required, that the source faded by $20 \%-50 \%$ in the $1.58 \mathrm{yr}(0.41 \mathrm{yr}$ in the quasar frame) between the two observations. The 1992 September flux is consistent with the $(4.8 \pm 0.40) \times 10^{-13}$ ergs $\mathrm{cm}^{-2} \mathrm{~s}^{-1}$ measured by the Einstein IPC in 1979 March (0.16-3.5 keV, $\alpha_{E}=0.5$, Wilkes et al. 1993). Since the shapes of the two spectra are similar, in spite of possible flux variations, we combined the two spectra to improve the signal-to-noise ratio.

The best-fit value of the absorbing column in the co-added spectrum $\left\{N_{\mathrm{H}}=[6.9(+3.5,-1.8)] \times 10^{20} \mathrm{~cm}^{-2}, 1 \sigma\right.$ error for two interesting parameters, $\alpha_{E}$ and $\left.N_{H}\right\}$, is not consistent with the Galactic value at a high confidence level ( $>99.9999 \%$, $\Delta \chi^{2}=45.2$, for one additional degree of freedom). A spectral fit with local absorption fixed at the Galactic value plus free absorption at $z=2.85$ yields an intrinsic $N_{\mathrm{H}}$ of $[0.86(+0.49$, $-0.28)] \times 10^{22} \mathrm{~cm}^{-2}$ and a slope $\alpha_{E}=0.61 \pm 0.22$.

PKS 0438-436 has a complex radio spectrum (Morton, Savage, \& Bolton 1978).

\section{4. $P K S 2126-158, z=3.27$}

This is another radio-loud quasar with a gigahertz peaked spectrum (O'Dea, Baum, \& Stanghellini 1991), with a clear break at $\sim 5 \mathrm{GHz}$ (observed frame, Peterson et al. 1982). It is bright in X-rays, with a PSPC flux of $3.7 \times 10^{-12} \mathrm{ergs} \mathrm{cm}^{-2}$ $\mathrm{s}^{-1}(0.1-2.5 \mathrm{keV})$. We observed the source twice, in $1991 \mathrm{May}$ and 1992 November, obtaining a similar count rate. The PSPC count rate of 0.17 counts $\mathrm{s}^{-1}$ is 1.9 times the 1979 Einstein IPC count rate of 0.09 ct s$^{-1}$ (Worrall \& Wilkes 1990), consistent with no variation between the two observations (see $\S 2.2$, ROSAT Mission Description 1991). [Also the HST snapshot survey found that PKS 2126-158 had $V=17.2 \pm 0.2$ on 1991 June 6 (Maoz et al. 1992), only 1 month after our ROSAT observation and consistent with the $V=17.3$ Palomar Sky Survey magnitude; Jauncey et al. 1978.]

The two PSPC spectra were very similar, as were the best-fit parameters in Table 2, and therefore we combined the two spectra together to improve the signal-to-noise ratio. The coadded spectrum is shown in Figure $1 d$ where a deficit of lowenergy counts can be seen similar to that in S5 0014+81 and PKS $0438-386$. The Galactic $N_{\mathrm{H}}$ toward PKS 2126-158 is only $4.85 \times 10^{20}$ atoms $\mathrm{cm}^{-2}$, so that excess absorption is again suggested. Indeed, while the spectral index is consistent with the mean spectral index found in low-redshift, radio-loud quasars, the best-fit value of the absorbing column $\left\{N_{\mathbf{H}}=\right.$ $[12.9(+7.2,-3.8)] \times 10^{20} \mathrm{~cm}^{-2}, 1 \sigma$ error for two interesting parameters, fit 1$\}$, is not consistent with the Galactic value at high confidence level $\left(>99.999 \%, \Delta \chi^{2}=28.9\right.$, for one additional degree of freedom, fit 2). $N_{\mathrm{H}}$ is significantly greater than the Galactic value in both observations (at the $99 \%$ and $99.99 \%$ confidence level, respectively). No other bright source is present in the PSPC image so that, unlike the case of PKS 0438-386 (Wilkes et al. 1992), we do not have an independent test against transient instrumental or local Galactic $N_{\mathrm{H}}$ peculiarities.
A spectral fit with local absorption fixed at the Galactic value plus free absorption at $z=3.27$ (fit 3) yields an intrinsic $N_{\mathrm{H}}$ of $[1.45(+1.20,-0.64)] \times 10^{22} \mathrm{~cm}^{-2}$ while not changing significantly the slope $\left[\alpha_{E}=0.52(+0.25,-0.21)\right]$. Worrall \& Wilkes (1990) also found evidence for absorption in excess of the Galactic value in PKS $2126-158$ at the $98.79 \%$ confidence level, implying that the absorption we see is not transient.

\section{5. $S 40636+680(z=3.18), P K S 2000-330(z=3.78)$}

For the two quasars with $\sim 60$ counts we investigated the possibility that they might also have additional absorbing column density. We did this by fixing the spectral index in the fit to the mean of the other four, and allowed the intrinsic absorbing column density to vary. The resulting fits are given in Table 2 .

With this prescription S4 $0636+680$ shows evidence for additional absorption beyond the Galactic value (at $98 \%$ confidence, $\Delta \chi^{2}=5.6$, for one additional degree of freedom), at a level similar to that of PKS 2126-158 and PKS 0438-436. Only for power-law slopes flatter than $\alpha_{E}=0.2 \quad(68 \%$ confidence) does the spectrum of S4 $0636+680$ become consistent with the Galactic column density. Such a flat slope would be rare. Of 13 quasars studied in this energy range by Ginga, Williams et al. (1993) found only one, PG 1416-129, with a slope this flat $\left[\alpha_{E}=0.10(+0.16,-0.21)\right]$, and the next flattest had $\alpha_{E}>0.4$. On average, flat spectrum radio core-dominated quasars have flat slopes (Shastri et al. 1993), but these two are predominantly steeper than $\alpha_{E}>0.4$. In contrast, S4 $0636+680$ has a steep high-frequency radio spectrum (Kühr et al. 1981) and is a strong GPS candidate (O'Dea et al. 1991).

The X-ray spectrum of PKS $2000-330$ is consistent with the Galactic absorption, but the rather large value of the Galactic column density and the limited number of counts prevent the detection in this spectrum of an additional column density similar to that detected in the other quasars. PKS 2000-330 is also a candidate GPS source in the list of O'Dea et al. (1991).

\section{DISCUSSION}

The ROSAT results reported here extend the study of quasar spectra to double the previous logarithmic range of luminosity and increase the range of redshift from about 0.3 to 3 . Although we have so far only four objects with determined spectra, we can set interesting limits on any change in mean $\mathrm{X}$-ray slope with luminosity or redshift.

The major, and unexpected, result of our observations is the discovery of significant absorption in a second (and possibly a third) high-redshift quasar. Similar absorption could have been detected in only four of the six high-redshift quasar spectra presented here. This high rate of detection implies that among $z=3$ quasars X-ray absorption is common, but not universal: the nondetection of absorption in one object out of four gives binomial bounds on the mean number of absorbers to $z=3$ of $0.4-0.9$ (90\% confidence). The study of high-redshift X-ray absorption allows us to investigate physical conditions in the intervening material between us and the quasar, and possibly in the quasar environment. We discuss each of these absorbing possibilities below, after first commenting on the derived spectral indices.

\subsection{No Change in $X$-Ray Slope with Luminosity or Redshift}

A redshift of three corresponds to a look-back time of $75 \%$ the age of the universe (for $\Omega_{0}=0 ; 67 \%$ for $\Omega_{0}=1$ ). During this time the quasar luminosity function shifted upward in luminosity by a factor $\sim 100$ in the optical (Boyle et al. 1987), 
and a factor $\sim 50$ in X-ray luminosity (Boyle et al. 1993). Spectral changes in the quasar continuum between local quasars and $z=3$ would seem inevitable. For example, in the standard scenario of accretion onto a black hole, a large change $(>100)$ in $L / L_{\mathrm{Edd}}$ for quasars is implied if it is assumed (admittedly naively) that the observed luminosity evolution is followed by individual quasars ${ }^{3}$ (i.e., assuming that they are rare but longlived). The $z=3$ quasars we have observed are $\sim 100$ times more luminous than those studied by Ginga, and so represent the early form of the Ginga quasars in the long-lived picture. Alternatively, if quasars are common but short-lived they will most likely remain near the Eddington luminosity and no spectral evolution need be expected. Changes in X-ray slope with redshift were first searched for by Canizares \& White (1989) using Einstein IPC data. The limited signal-to-noise ratio available prevented any strong constraints being placed. The ROSAT data allow us to revisit this question.

Previously published X-ray spectral slopes for low-redshift quasars are plotted as open symbols in Figures $5 a$ and $5 b$ as a function of the redshift and of the $2-10 \mathrm{keV}$ luminosity $L_{2-10}$. These slopes are 1.7-18 keV spectral indices from Ginga observations (Williams et al. 1992). The errors on the indices plotted are $\pm 1 \sigma$ for one interesting parameter, as listed in Williams et al. (1992). The Ginga data appear to show a flattening with redshift but, as Williams et al. show, this is a secondary effect of the well-known difference in mean slope between radio-quiet and radio-loud quasars (Wilkes \& Elvis 1987). This can be seen by comparing the two types (radio-quiet are plotted as circles while radio-loud are plotted as squares). The observed range of luminosities for radio-loud quasars alone is only one and a half decades in Williams et al. (1992), so it is unsurprising that no dependence is seen. The ROSAT data now extend this analysis to higher luminosities and redshifts.

${ }^{3}$ Note that, with these assumptions, $\dot{m}$ decreases toward the present by a factor 50-100 and, since $M$ can only increase, $L / L_{\text {Edd }}$ must decrease toward the present.

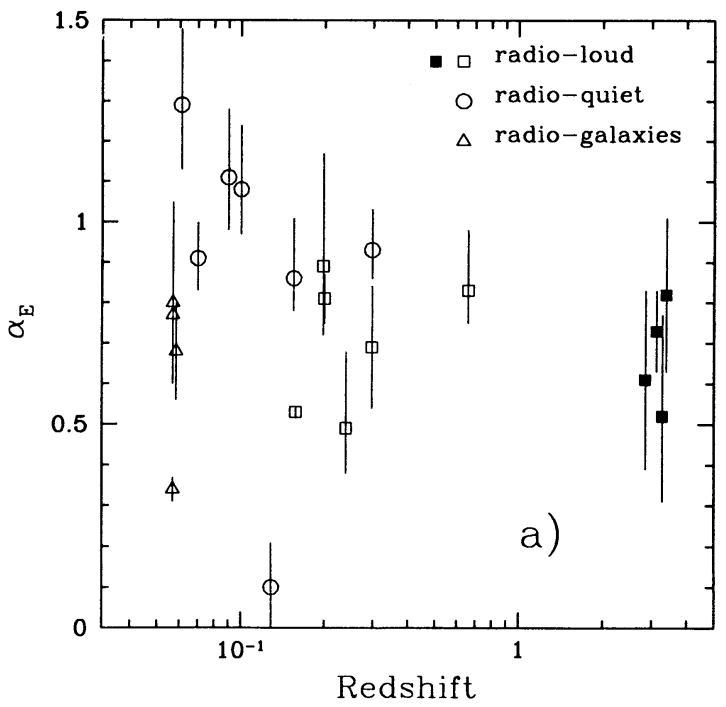

FIG. $5 a$
The ROSAT PSPC spectral indices for our four quasars are plotted as filled symbols in Figures $5 a$ and $5 b$. For the two objects which show intrinsic absorption we have plotted the spectral index obtained allowing both intrinsic $N_{\mathrm{H}}$ and $\alpha_{E}$ to vary, while for the other two objects we plot the spectral index obtained fixing $N_{\mathrm{H}}$ to the Galactic value. The uncertainties on the PSPC slopes are comparable with those of Ginga. The mean PSPC spectral index for the four high-redshift, radioloud quasars is $0.71 \pm 0.08$, well within the $90 \%$ confidence range of Williams et al. (1992) for radio-loud quasars of 0.560.86 . Figures $5 a$ and $5 b$ can also be extended to lower luminosities and redshifts using Ginga and EXOSAT results on broad-line radio galaxies. Four such radio galaxies have X-ray spectra in the same $\sim 1-10 \mathrm{keV}$ rest frame energy range (references are given in the figure caption) and these are plotted in Figures $5 a$ and $5 b$ as triangles (3C 390.3 has two measurements). The mean spectral index of these four radio galaxies is $0.61 \pm 0.02$, which is also consistent with the spectral indices found both in the Ginga and ROSAT radio-loud quasars.

No change in the mean rest frame $1-10 \mathrm{keV}$ spectral index greater than 0.3 is detected (at the $99 \%$ confidence level) in either plot. There is no significant correlation in our data between the X-ray spectral indices of radio-loud quasars and their redshift or luminosity (the probability for a correlation is $36 \%$ and $30 \%$, respectively). This lack of spectral evolution must begin to set limits on models for quasar X-ray production. Short lifetime models for quasars would seem to be favored. Since we have no radio-quiet quasars as yet we can make no statement on their X-ray spectral evolution.

\subsection{Consequences of the Lack of Absorption toward Q0420-388}

In Q0420-388 our limit on X-ray absorption is significantly lower than in PKS $0438-436$ and PKS 2126-158, although it could still be as large as a few $10^{21}$ atoms $\mathrm{cm}^{-2}$. This absence of absorption toward one quasar shows that the observed

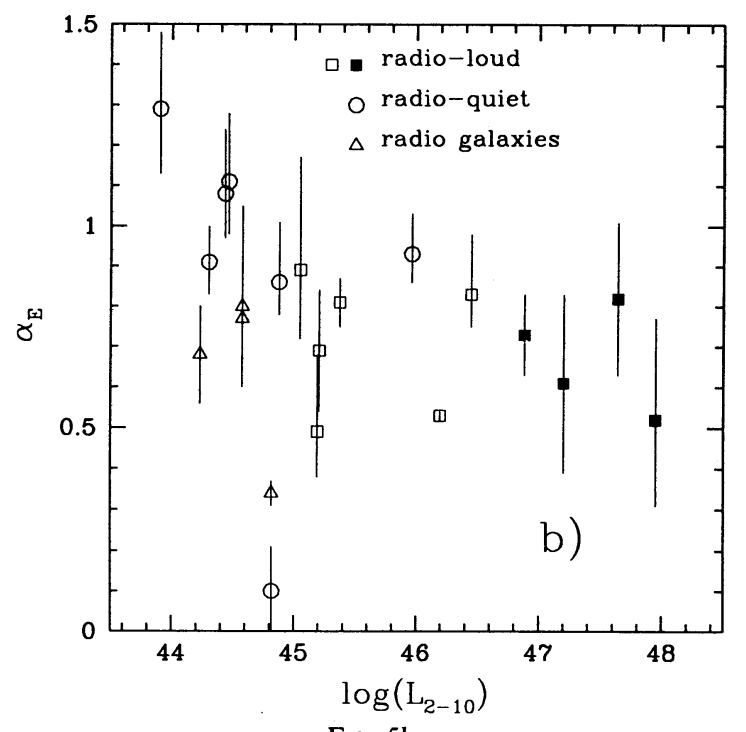

Fig. $5 b$

FIG. 5.-X-ray energy spectral index in the $\sim 1-10 \mathrm{keV}$ emitted frame vs. (a) redshift, (b) luminosity. Squares represent radio-loud quasars, circles radio-quiet quasars, and triangles radio galaxies. The ROSAT PSPC spectral indices for the four $z \sim 3$ quasars are plotted as filled symbols. The other points (open symbols and triangles) are Ginga results reported by Williams et al. (1992) for the quasars and by various authors for the radio galaxies (3C 445, Pounds 1990; Pictor A, Singh, Rao, \& Vahia 1990; 3C 382, Kaastra, Kunieda, \& Qwaki 1991; 3C 390.3, Inda et al. 1993). 
absorption is not universal and so not due to a uniform intergalactic medium. In fact, the limit on the X-ray absorption can be turned into a limit on the density of such a medium analogous to the Gunn-Peterson test for Ly $\alpha$ (Gunn \& Peterson 1965; Peebles 1993). The X-ray version of this test remains sensitive even for high temperatures $\left(10^{5-6} \mathrm{~K}\right)$ where the Ly $\alpha$ test loses power (Shapiro \& Bahcall 1980) as hydrogen becomes ionized. In this way it complements both the Ly $\alpha$ test and the COBE limits on the Compton " $y$-parameter," which limits the density of a hot $\left(>10^{7} \mathrm{~K}\right)$ intergalactic medium (IGM). To apply the X-ray Gunn-Peterson test is, however, complicated both by the assumptions about the thermal history and metallicity of the IGM, and by the limited spectral resolution of proportional counters. We thus defer discussion of this limit to a later paper.

\subsubsection{The Ionization State of Damped Ly $\alpha$ Systems}

Damped Ly $\alpha$ systems have large column densities of neutral hydrogen compared to other types of quasar absorption lines. They are often considered to be due to extended disks of galaxies at an early stage of evolution (Wolfe 1988). The physical conditions of the absorbing gas are poorly determined from absorption line studies which sample only a few ions (Lanzetta et al. 1991, and references therein). A basic parameter is the ionization state of the gas. In damped Ly $\alpha$ systems the fraction of neutral hydrogen is not easily determined. If the ionization state were determined the presence of an ionizing background radiation could be used to set bounds on the density, size, and mass of the absorbing material. Since the X-ray absorption cross section is relatively insensitive to ionization and depletion (Morrison \& McCammon 1983), X-ray measurements give a total column density and so can limit the ionization state of Ly $\alpha$ systems.

Q0420-388 has two damped Ly $\alpha$ systems in its optical spectra, and our limit on X-ray absorption toward this quasar allows us to provide a direct observational limit on their state. The absorbers (which are close to the quasar emission-line redshift, at $z \sim 3.08$; Atwood, Baldwin, \& Carswell 1985) have a total $N(\mathrm{H} \mathrm{I})=5 \times 10^{19} \mathrm{~cm}^{-2}$. Our upper limit on any absorption from them is $N(\mathrm{H})=3.4 \pm 3.3 \times 10^{21}$ atoms cm $\mathrm{cm}^{-2}$. This gives a lower limit on the neutral to total hydrogen ratio, $N(\mathrm{H} \mathrm{I}) / N(\mathrm{H})>0.004(3 \sigma)$, which is quite low. For nonsolar metal abundances, which may be $1 / 10$ or lower in damped Ly $\alpha$ systems, the implied $\mathrm{X}$-ray hydrogen column density can increase by up to a factor of $\sim 4(\S 2$ Fig. 3$)$, in which case $N(\mathrm{H} \mathrm{I}) / N(\mathrm{H})>0.001$.

Another limit to the neutral fraction of hydrogen in these systems comes from the Atwood, Baldwin, \& Carswell detection of Si II in the damped Ly $\alpha$ systems, with a total column of $N(\mathrm{Si}$ II $)=6.3 \times 10^{15}$ atoms $\mathrm{cm}^{-2}$. Assuming a solar abundance $(\mathrm{Si} / \mathrm{H})=10^{-4.43}$ (Morrison \& McCammon 1983), the Si column density corresponds to $N(\mathrm{H})=1.7 \times 10^{20}$ atoms $\mathrm{cm}^{-2}$, so $N(\mathrm{H} \mathrm{I}) / N(\mathrm{H})<0.27$. This is an upper limit since there can be $\mathrm{Si}$ in other ionization states which are not currently detectable, and $\mathrm{Si}$ may have lower abundance, thus increasing the implied $N(\mathrm{H})$.

Lanzetta et al. (1991) find that the integrated amount of neutral material in all damped Ly $\alpha$ systems can account for $\Omega_{0}=1$, so that statistically the amount of ionized material cannot exceed this by more than a factor of a few. In contrast, our X-ray measurement is a direct limit for a particular system.

The above neutral fraction limit can be converted into a limit on size and density, given an assumed background ionizing radiation field $J(912 \AA)$. As an example, we have taken
$J(912 \AA)=10^{-21}$ ergs $\mathrm{cm}^{-2} \mathrm{~s}^{-1} \mathrm{~Hz}^{-1} \mathrm{Sr}^{-1}$ (Lu, Wolfe, \& Turnshek 1991; Bajtlik, Duncan, \& Ostriker 1988) and solar abundances. This leads only to a weak limit on the size and density of the absorbing material, less than $3 \mathrm{Mpc}$, and greater than $10^{-3} \mathrm{~cm}^{-3}$ (Fig. 6).

The value of $J(912 \AA)$ used is a lower limit to the ionizing flux experienced by the absorber. The damped systems are close to the " proximity effect" Strömgren sphere of the quasar, so the UV radiation from the quasar may be as important as the metagalactic flux depending on the latter's value. The clouds are also expected to be in a galactic environment and so are likely to be subject to a strong stellar radiation field. Finally, unlike the Ly $\alpha$ forest clouds, the damped systems are unlikely to be solely photoionized. Qualitatively, all these factors move the limits on size and density toward smaller, denser clouds.

The size limit also depends sensitively on the X-ray column density limit. For a limit on $N_{\mathrm{H}}<10^{21}$ atoms $\mathrm{cm}^{-2}$ this limit would become less than $25 \mathrm{kpc}$ (Fig. 6), and so would constrain the absorber to no more than galaxy-like dimensions. Such a limit should be readily attainable with soft X-ray instrumentation (although the PSPC itself is not likely to be available for long enough for such an observation to be performed).

\subsection{High-Redshift X-Ray Absorption}

The appearance of large amounts of absorption in the X-ray spectra of high-redshift quasars is surprising in two ways. First, these quasars have strong blue ultraviolet continua that give no hint of large amounts of obscuration; second, heavy obscuration has been rarely reported in high X-ray luminosity AGN at low redshift. We first discuss these two points, and then go on to consider possible intervening and intrinsic sites for the absorbers.

The lack of ultraviolet reddening has implications for the amount and distribution of dust in the absorber. For standard Milky Way values of the interstellar dust-to-gas ratio an $\mathrm{H} \mathrm{I}$ column density of $10^{22}$ atoms cm $\mathrm{cm}^{-2}$ corresponds to $A_{V} \sim 4$. As Bechtold et al. (1994) will discuss, a value of $A_{V}>0.5$ is highly unlikely to be affecting the ultraviolet spectrum of these

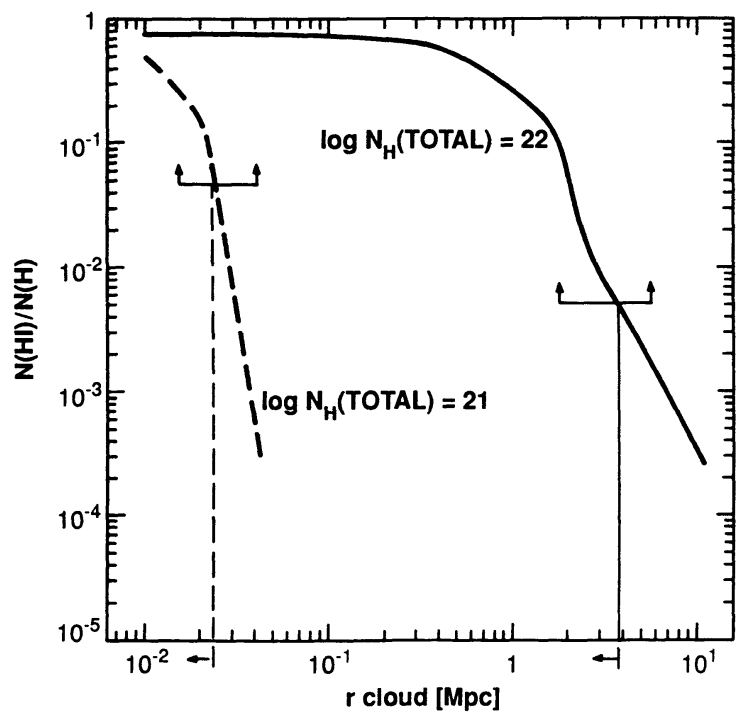

Fig. 6.-Physical size of the damped Ly $\alpha$ system in Q0420 - 388 vs. ionized fraction for a PSPC-derived total column density of less than $10^{22}$ atoms $\mathrm{cm}^{-2}$ (solid line). The nonlinear dependence on the X-ray-derived column density is shown with the dashed line that gives the limits in the hypothetical case of a limit of less than $10^{21}$ atoms $\mathrm{cm}^{-2}$ (dashed line). 
quasars. There are several possibilities to explain this apparent contradiction: (1) The metal abundances at $z=3$ could be much reduced so that a large $N_{\mathrm{H}}$ does not correspond to a large metal, and hence dust, column; (2) the dust-to-gas ratio in the absorber may be much lower than in the Milky Way; (3) the dust in the absorbers may contain only large grains that do not cause reddening (see, e.g., Laor \& Draine 1993); (4) the absorber may have a geometry that obscures only the X-ray source and not the ultraviolet source, in which case it must originate close to the active nucleus.

We argue below that (4) is unlikely. In cooling flows, a possible site for the absorber (see $\S$ 3.3.2), X-ray sputtering may well destroy small grains (see White et al. 1991, and references therein), so that (3) has some physical justification. The dustto-gas ratio in damped Ly $\alpha$ systems is much debated because of its importance to quasar visibility at high redshifts, and its importance for early galaxy evolution (see Fall \& Pei 1993 and references therein). Pei, Fall, \& Bechtold (1991) show that, for Milky Way-type dust, this ratio must be no more than $1 / 10$ the Milky Way value. Others have suggested that the metal abundances in damped Ly $\alpha$ systems is no more than $1 / 10$ solar (Meyer \& Roth 1990; Pettini et al. 1990; Turnshek et al. 1989). Possibilities (1) and (2) are therefore plausible for intervening systems.

$\mathrm{X}$-ray absorption in quasars of high X-ray luminosity is highly unusual, column densities less than $5 \times 10^{20}$ atoms $\mathrm{cm}^{-2}$ being normal (Wilkes \& Elvis 1987; Kruper, Urry, \& Canizares 1990; Williams et al. 1992). In Figures $7 a$ and $7 b$ we show a plot of excess absorption against redshift and X-ray luminosity compiled from Ginga, Einstein, and ROSAT measurements. (References are given in the figure legend.) From Figure 7 it is clear that at low $\mathrm{X}$-ray luminosities $\left(<10^{44} \mathrm{ergs}\right.$ $\mathrm{s}^{-1}$ ) large X-ray column densities are common in AGN (Lawrence \& Elvis 1982; Turner \& Pounds 1989), while above $10^{44}$ ergs s $^{-1}$ a clear drop in the number of absorbed systems can be seen. Note that this drop is not due to insensitivity to column density by Ginga, or Einstein, nor is it due just to small number statistics. (There are more than 20 quasars in this range. The binomial probability of finding three out of four absorbed when one in 20 is expected is 0.0005 ; both Ginga at low redshift, and ROSAT at high redshift, are sensitive to the same emitted energy range.) The high-redshift ROSAT results reverse this trend. Is a new mechanism needed to produce the absorption detected at high $z$ and/or luminosity, or can known low $z$ absorbers suffice? First we consider the two previously suggested sites for absorption in AGN-the broad emission line region and jets. We find that these are unlikely to apply to the high-redshift quasars.

Since the absorption in low-luminosity AGN is time variable it is normally associated with the environs of the broad emission-line region. That the cases of absorption seen above a luminosity of $10^{44}$ ergs $\mathrm{s}^{-1}$ at low $z$ are "warm" (i.e., partially ionized, see Fig. 7, open circles) suggests that this site becomes too ionized by the continuum to be effective at higher luminosities. An evolutionary change in the broad emission-line region could be invoked, but the emission lines of quasars show no striking changes with redshift (Schneider, Schmidt, \& Gunn 1989).

Cool material in a relativistic jet may appear implausible, yet X-ray absorption is seen in several BL Lac objects (Canizares \& Kruper 1984; Madejski et al. 1991) and in the optically violent variable (OVV) NRAO 140 (Marscher 1988). Since PKS $0438-436$ is a strong, core-dominated radio source it is likely to be relativistically beamed toward us, leading Wilkes et al. (1992) to suggest that the absorbing material may be located within the radio-emitting jet. However, neither PKS 0438-436 nor PKS 2126-158 are clearly blazars, since neither has polarization that is convincingly above the $3 \%$ threshold that is normally used to indicate blazar-like activity (Impey \& Tapia 1990). For PKS 0438-436 Impey \& Tapia (1990) report $4.7 \% \pm 1.0 \%$, but Fugmann \& Meisenheimer (1988) find only $1.6 \% \pm 0.5 \%$. Wills et al. (1992) report an optical polarization of only $1.7 \% \pm 0.9 \%$ for PKS $2126-158$.

More tellingly, PKS 2126-158 and S4 $0636+680$ are GPS radio sources. Beaming is not believed to be important in GPS sources because of their low radio polarization, steep high-
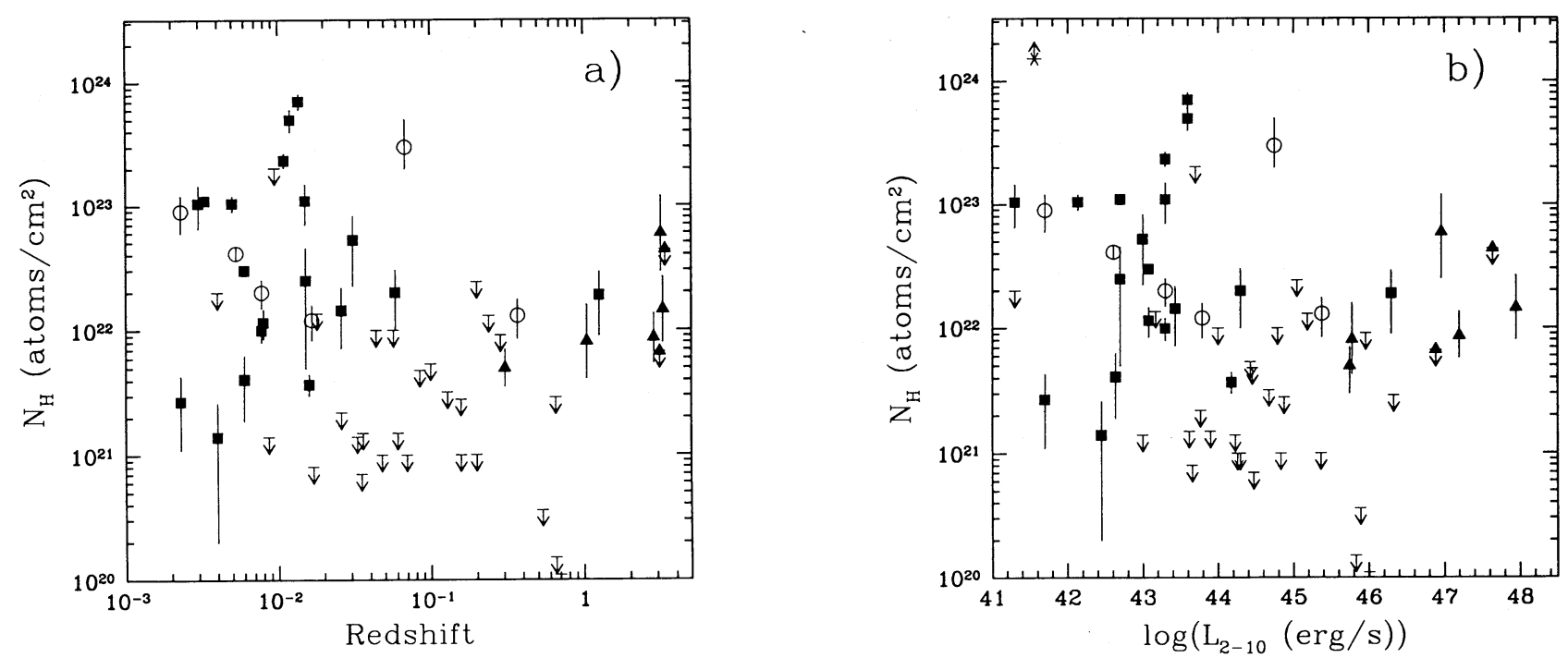

Fig. 7.-Absorbing X-ray column density vs. redshift (a), 2-10 keV luminosity. (b) The upper limits and solid squares below $z=0.3$ are Ginga measurements taken from Awaki (1992) and Nandra (1992). The upper limits and solid squares at $z>0.3$ are Einstein IPC measurements (Wilkes \& Elvis 1987; Marscher 1988). Triangles indicate ROSAT measurements and upper limits from this paper and from Allen \& Fabian (1992). Open circles indicate likely "warm" absorbers (from Pan et al. 1990; Fiore et al. 1992, 1993; Turner et al. 1992; Nandra \& Pounds 1992). 
frequency spectra, and lack of strong variability (O'Dea et al. 1991).

Since beaming is probably unimportant, the large derived X-ray luminosities of the GPS quasars PKS 0420-388 and PKS 2126-158 are thus likely to be true $4 \pi$ values (Phillips \& Mutel 1982). Such luminosities imply extreme masses $\left(\sim 10^{10-11} M_{\odot}\right)$ for the presumed massive black holes at their centers, and predict strong limits on any $\mathbf{X}$-ray variability $(\sim 1$ yr quasar frame, $4 \mathrm{yr}$, observed frame).

The reappearance of absorption at luminosities above $10^{46}$ ergs $\mathrm{s}^{-1}$ and/or at redshifts greater than one in the ROSAT PSPC data suggests that a different mechanism may be coming into play. Since the broad emission-line region and jets are unlikely, we are forced to examine other sites, farther from the nucleus. We next consider the possibility that the absorption is unconnected with the quasar, merely lying along the line of sight; and then go on to consider an origin in the quasar environment on a galaxy or cluster of galaxies scale.

\subsubsection{Intervening Absorbers: Damped Ly $\alpha$ Systems?}

Since the PSPC energy resolution does not allow us to determine the redshift of the absorption, it may also lie anywhere along the line of sight between our galaxy and the quasar. Absorption would then be seen primarily in high $z$ quasars simply because they have a longer path length along which an absorber might lie. The path length for absorption for a uniformly distributed population of absorbers is given by the coordinate $X$ (in units of $c / H_{0}$, Wagoner 1967; Bahcall \& Peebles 1969) which allows for the increased crowding of objects as $z$ increases $\left\{X=0.5\left[(1+z)^{2}-1\right], \Omega_{0}=0 ; X=\right.$ $\frac{2}{3}\left[(1+z)^{3 / 2}-1\right], \Omega_{0}=1$. $\}$ Figure 8 shows the absorption path length, $X$, toward our $z=3$ quasars PKS 0438-436 and PKS $2126-158$. The lack of absorption toward quasars at $z \sim 0.3-$ 0.4 (Wilkes \& Elvis 1987) excludes only a small region near the origin. At $z=3 X$ is 22 times larger than at $z=0.3$ (for $\Omega_{0}=0$, 15 times for $\Omega_{0}=1$ ), so a $5 \%$ chance of an intervening system in the low-redshift objects becomes close to unity by $z=3$, if there is no evolution in the mean free path for absorption. This makes an intervening explanation seem quite natural.

In the context of intervening absorbers the sensitivity of $\mathrm{X}$-ray absorption to higher $Z$ elements makes the abundances and the ionization state of the absorbers critical to an assessment of the probability that a particular absorption system causes the X-ray absorption. The ionization states are often poorly known from optical and ultraviolet absorption lines since these lines sample only a limited number of elements and ions. Conversely, given a redshift, X-ray absorption data can be used to determine or limit the ionization fraction (as in $\S 3.2$ ).

There are four major classes of candidate intervening absorbers in the optical spectra of quasars (Sargent 1988). In order of increasing column density they are: Ly $\alpha$ forest lines $\left(\sim 10^{12-16}\right.$ atoms $\left.\mathrm{cm}^{-2}\right)$; Lyman limit systems $\left(\sim 10^{>17}\right.$ atoms $\left.\mathrm{cm}^{-2}\right)$; metal line systems $\left(\sim 10^{>16}\right.$ atoms $\left.\mathrm{cm}^{-2}\right)$; and damped Ly $\alpha$ systems $\left(\sim 10^{19-21}\right.$ atoms $\left.\mathrm{cm}^{-2}\right)$. If sufficiently highly ionized, absorbers of almost any type might produce the observed X-ray absorption. However, the damped Ly $\alpha$ absorbers are the most straightforward to associate with the X-ray absorption due to their large $N\left(\mathrm{H}_{\mathrm{I}}\right)$ values (Sargent 1988; Wolfe 1988).

Neither of the well-determined X-ray absorbers shows any intervening damped Ly $\alpha$ system at $z>1.88$ (Morton, Savage, \& Bolton 1978; Sargent, Steidel, \& Boksenberg 1990; Lan-

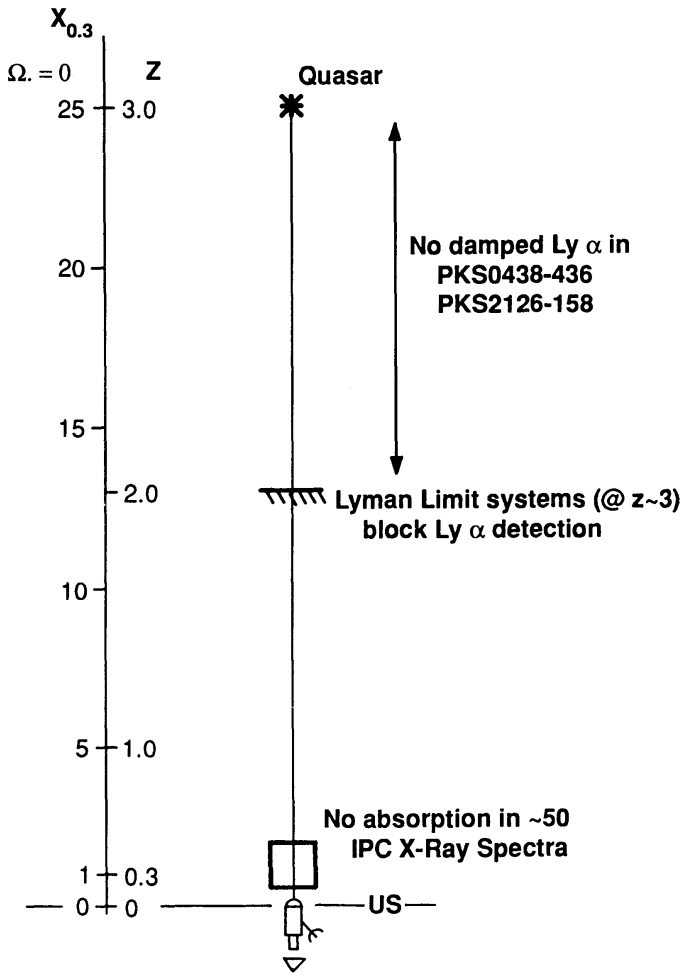

FIG. 8.-Possible locations for damped Ly $\alpha$ absorbers toward PKS $0438-436$ and PKS $2126-158$ as a function of the path length for absorption, $X$. Previous limits from studies of quasars at $z \sim 0.3-0.4$ constrain the absorption very little. No damped Ly $\alpha$ systems are found at $z>1.88$ in either quasar, which rules out half of the available path length for absorption. The presence of Lyman limit systems cuts out all information about $\operatorname{Ly} \alpha$ absorbers at $z<1.88$ in both quasars.

zetta, Turnshek, \& Wolfe 1987). ${ }^{4}$ This eliminates half of the available absorption path length for damped Ly $\alpha$ absorption systems (Fig. 8). Below this redshift Lyman limit systems in both quasars cut out all emission, so no other absorption systems can be detected. The Lyman limit system in PKS $2126-158$ has a column density of $N(\mathrm{H} \mathrm{I})>2-3 \times 10^{17}$ atoms $\mathrm{cm}^{-2}$ (Sargent et al. 1990) and has no associated Ly $\alpha$. PKS $0438-436$ has not been studied for absorption systems. If this were the origin of the absorption we see it implies $N(\mathrm{H} \mathrm{I})$ / $N(\mathrm{H})<3 \times 10^{-5}$.

Given the number density of absorbers per unit redshift, $d N / d z$, of $\mathrm{Ly} \alpha$ absorption systems with large $\mathrm{H}$ I column density we can derive the probability of finding two such absorbers at $z<1.88$ toward two out of three quasars:

$$
\frac{d N}{d z}=\sigma \rho_{0}(1+z)\left(1+2 q_{0} z\right)^{-1 / 2},
$$

where $\sigma$ is the cross section of the absorbers, and $\rho$ is their space number density. Empirical fits to $d N / d z$ are usually made assuming: $d N / d z=A_{0}(1+z)^{\gamma}$, where $A_{0}$ and $\gamma$ are constants. For $\Omega_{0}=0$ and no evolution $\gamma=1$, while for $\Omega_{0}=1$ and no evolution $\gamma=\frac{1}{2}$. Lanzetta et al. (1991) have two samples for which they find: $A_{0}=0.035, \gamma=1.2 \pm 1.7 ; A_{0}=0.163$, $\gamma=0.3 \pm 1.4$. Errors in $A_{0}$ and $\gamma$ are highly correlated, so for

${ }^{4}$ A possible $\mathrm{NaD}$ line in the spectrum of PKS $2126-158$, which would imply extremely low ionization and so a large column density, is not confirmed in a spectrum taken by one of us (J. B.) 
simplicity we assume that the major uncertainty is in $\gamma$. Integrating these empirical fits over $0<z<1.88$, assuming no evolution, gives an expected number of systems of 0.147 and 0.371 , respectively. Within the uncertainties the predicted numbers of absorbers are consistent with the mean of 0.4-0.9 $\mathrm{X}$-ray absorbers per $z=3$ quasar that we find. For $0<z<0.5$ these numbers are much smaller, 0.023 and 0.087 , so that not seeing absorbers in the X-ray spectra of low-redshift quasars (Wilkes \& Elvis 1987) is also consistent with an intervening explanation.

The X-ray case is somewhat more complicated than that for Ly $\alpha$ since $\sigma$ is energy and abundance-dependent. Figure 4 shows that for a given $N_{\mathrm{H}}$ the cutoff in the X-ray spectrum declines as $z_{\text {absorber }}$ increases, roughly as $(1+z)$. Figure 3 shows primordial material has a $\sigma$ about $\frac{1}{4}$ that of solar material. The dependence of $\sigma$ on $z$ and abundances makes it understandable how the high $z$ damped Ly $\alpha$ absorber in Q0420 - 388 cannot strongly affect the PSPC spectrum, while lower $z$ systems with the same $N(\mathrm{H} \mathrm{I})$ would produce an X-ray cutoff.

There are three unknowns: the evolution of high column density absorbers toward low redshift; the ionization state of these absorbers; and their abundances. Lower space densities for nearby absorbers would make an intervening explanation less likely. The space density of damped Ly $\alpha$ systems below $z \sim 2$ has not been studied to date because of the limited path length to any one object, so that large samples are needed to make strong statements. A decline in numbers toward the present is allowed. Models that ascribe the damped Ly $\alpha$ systems to halos of protogalaxies must have a strong reduction in their space densities toward low redshift (Wolfe 1988). The use of $z=2$ densities at lower redshifts thus requires a large extrapolation. A higher ionization level would enable absorbers with lower neutral $N_{\mathbf{H}}$ to produce the observed $\mathrm{X}$-ray column, thus raising the total number of absorbers available. To produce an X-ray column density of $1-5 \times 10^{21}$ atoms $\mathrm{cm}^{-2}$, an absorber with solar metal abundances and $N(\mathrm{H} \quad \mathrm{I})=1-5 \times 10^{20}$ atoms $\mathrm{cm}^{-2}$ would need $N\left(\begin{array}{ll}\mathrm{H} & \mathrm{I}\end{array}\right) /$ $N(\mathrm{H})=10^{-1}$. This is within our own limit on $N(\mathrm{H} \mathrm{I}) / N(\mathrm{H})$ for $\mathrm{Q} 0420-388(\S 3.2)$. Since $d N / d z$ rises with a slope of 1.8 to lower $N(\mathrm{H} \mathrm{I})$ even a factor 4 higher ionization fraction would give 12 times the number of expected absorbers.

Thus for both quasars intervening damped Ly $\alpha$ systems can plausibly produce the X-ray absorption. However, the argument is statistical, and it is worthwhile to consider alternative explanations.

\subsubsection{Intrinsic Absorption: Intracluster Gas at $z=3$ ?}

The large look-back time to redshift 3 allows plenty of scope for evolutionary changes in the host galaxy and in the larger scale environment. Most of the potential changes are not yet theoretically well-developed, however. An origin related to cluster environment is plausible, since all the observed quasars are radio-loud, and at $z>0.7$ radio-loud quasars tend to be found in rich galaxy environments (Yee \& Green 1984; Ellingson, Yee, \& Green 1991). In hierarchical biased cold dark matter (CDM) scenarios clusters of galaxies are predicted to form at the peaks of initial density fluctuation at $z \gtrsim 2$ (Kaiser 1984; Davis et al. 1985). Optical searches for distant clusters reveal several dense cores which are already relaxed (i.e., have high-velocity dispersions $\left\langle v^{2}\right\rangle^{1 / 2} \gtrsim 10^{3} \mathrm{~km} \mathrm{~s}^{-1}$ and nearly homogeneous galactic populations) at $z \gtrsim 0.7$ (Gunn 1990; Ellis 1992), thus suggesting the presence of hot and dense gas at those $z$.
The presence of GPS sources in our sample suggests an intriguing possibility for the site of the absorbers in these highredshift quasars. GPS sources are unusually compact $(\sim 10$ milliarcsec, $\sim 100$ pc at $z=3$; Pearson \& Readhead 1984). This compactness likely implies a dense surrounding medium to confine the radio source. A cooling flow around the quasar provides a plausible mechanism and could also explain the absorbing columns that we have detected.

The pressures needed to produce the low-frequency cutoff in the radio spectrum due to free-free absorption are $\sim 10^{8} \mathrm{~cm}^{-3}$ $\mathrm{K}$ (O'Dea et al. 1991). These are similar to the pressures in an X-ray cooling flow on a quasar (Fabian \& Crawford 1990). Thermal confinement of the GPS sources had been considered unlikely by O'Dea et al. (1991) because the amount of cold $\left(T \sim 10^{4} \mathrm{~K}\right)$ material needed is excessive. The presence of gas at X-ray temperatures would remove this problem and would tie together two key properties of GPS sources: confinement and low-frequency cutoffs. A cooling flow could also lead to a synchrotron self-absorption cutoff by keeping the radio source unusually compact. Neutral hydrogen has been detected in absorption against a radio galaxy at $z=3.4$ (B2 0904+343; Uson, Bagri, \& Cornwell 1991) with an $\mathrm{H}$ I column density of $\sim 4.4 \times 10^{18} T_{s}$ atoms $\mathrm{cm}^{-2}$, where $T_{s}$ is the spin temperature of the $\mathrm{H} \mathrm{I}$. $T_{s}$ could have a value of $\sim 10^{4}$ (Uson et al. 1991), so the total hydrogen column could be of the correct order of magnitude to be related to our X-ray absorbers.

At low redshifts $\mathrm{X}$-ray absorption from material associated with cooling flows has been detected with column densities up to a few $10^{21}$ atoms $\mathrm{cm}^{-2}$ (White et al. 1991), within a factor of a few as large as those seen in PKS 0438-436 and PKS $2126-158$. These column density values have been obtained assuming an uniform absorbing screen. The absorbing gas, however, is likely to be clumpy and mixed with the hot emitting gas. Active nuclei are small so that absorption toward them could well pass through only one cloud, and so record somewhat larger $N_{\mathrm{H}}$ values than the averaged values provided by the extended hot gas in a cluster core. There is consistency with the low-redshift AGN seen at the centers of cooling flows which do in fact have similarly large X-ray column densities (NGC 1275, Fabian et al. 1981; M87, Schreier et al. 1982; Cygnus A, Arnaud et al. 1987), as do, however, many AGN not in cooling flows. Thus the X-ray absorption we see in highredshift quasars may represent the detection of intracluster material at $z \sim 3$.

The fact that our quasars were selected to be among the most luminous known could bias us toward objects in strong cooling flows. Fabian \& Crawford (1990) have pointed out that the quasar continuum emission will Compton cool the material in a cooling flow, and so may increase the quasar accretion rate in a feedback that enhances the quasar luminosity. The most luminous quasars may thus be found in the strongest cooling flows.

One candidate GPS quasar, Q0420-388, does not show excess absorption. This does not in itself constitute evidence against the absorption in the high $z$ quasars being related to cooling flows. White et al. (1991), with better sensitivity to absorbing column densities than in our data, find excess absorption in only 12 out of 21 clusters. None of the three quasars with X-ray absorption has a damped Ly $\alpha$ or Lyman limit systems near the quasar emission redshift (Sargent et al. 1990; Morton et al. 1978), although PKS 0438 - 436 does have three narrow Ly $\alpha$ absorption lines superposed on the Ly $\alpha$ emission line. If the X-ray absorber is at $z(\mathrm{em})$, it must be 
highly ionized $\left[N(\mathrm{H} \mathrm{I}) / N(\mathrm{H})<10^{-5}\right]$. The quasar continuum will photoionize a region around the quasar that could well encompass the whole cooling flow region.

The X-ray continuum emission from any putative cluster or cooling flow is not likely to be a strong contaminant of the quasar spectrum. The highest X-ray luminosity known from a cluster of galaxies is $6.4 \times 10^{45} \mathrm{ergs} \mathrm{s}^{-1}(2-10 \mathrm{keV})$ for A2163 (Arnaud et al. 1992). This extreme cluster would contribute no more than $10 \%$ to the flux of Q0420-388, or $0.7 \%$ of PKS $2126-158$. An arcsecond resolution X-ray image would be able to directly resolve such cooling flows.

A cooling flow is not the only possible site for intrinsic absorption due to a cluster. Uson et al. (1991) also found extended $\mathrm{H}$ I emission at the same redshift as the absorption but $33^{\prime}$ away. This emission requires a large mass, and narrow line width implies a nonvirialized mass near turnaround (i.e., just separating from the Hubble flow), possibly a Zel'dovich pancake (Uson et al. 1991), or cluster of galaxies (Subramanian \& Swarup 1992). The H I column density of this "protocluster" is similar to that seen in our quasar X-ray spectra at similar redshifts, and an identification of such material with our absorbers may imply significant metal abundances in the "protocluster" material. Subramanian \& Swarup associate this $\mathrm{H}$ I with that of the damped Ly $\alpha$ systems, so that the same statistics used above apply to this explanation. However the lack of damped Ly $\alpha$ systems near the emission redshift in any of the high $z$ quasars with evidence for X-ray absorption, mentioned above, limits the ionization state and/or geometry of the " protocluster."

\section{4. $\mathrm{Fe}-K$ Lines}

Low-redshift broad emission line AGN emit strong Fe-K $6.4 \mathrm{keV}$ fluorescence lines (equivalent width of $50-300 \mathrm{eV}$, Nandra, Pounds, \& Stewart 1991). In addition, if the $z \sim 3$ quasars are surrounded by dense hot gas, they will show strong $\mathrm{Fe}-\mathrm{K}$ 6.5-7 keV lines (1000 eV equivalent width, with respect to the thermal continuum), and if the absorption of X-rays is due to intracluster gas, this will also produce an additional fluorescence line. The limits we derive for the $\mathrm{Fe}-\mathrm{K} 6.4 \mathrm{keV}$ fluorescence indicate that with moderately improved sensitivity iron $\mathrm{K}$ lines measurements of high-redshift quasars will begin to constrain models. The Gas Scintillation Proportional Counters and the CCDs which will be on board Asuka, $S A X$, and SPECTRUM $X-\gamma$ will probably be capable of detecting these lines, since the lines will fall at $1-2 \mathrm{keV}$ where all these missions have their greatest effective area.

\section{CONCLUSIONS}

We have presented X-ray spectral data for six $z=3$ quasars. Three of them show evidence for excess absorption along the line of sight above the Galactic value at $\sim 10^{22}$ atoms cm ${ }^{-2}$ (at the quasar redshift, for solar abundances). In two others such a column could not be detected due to the large Galactic column density. Only one quasar clearly has a lower level of absorption. We conclude that X-ray absorption is a common, but not universal, property of high-redshift, radio-loud quasars. The UV continua of these quasars show no sign of strong absorption.

Two explanations are at present equally plausible, one intervening and one intrinsic. If the detected X-ray absorption is due to intervening systems, them damped Ly $\alpha$ absorbers at $0.3<z<1.9$, are the prime candidates. Damped Ly $\alpha$ absorbers can have sufficient column density, and may have sufficient space density. However, this requires an extrapolation because the density of damped Ly $\alpha$ systems at redshifts below 2 is unknown.

If the absorption is intrinsic it is not likely to be associated with a jet, nor with the broad emission-line region. A possible site for the X-ray absorption lies in cold material in intracluster gas - for example a cooling flow-around the quasars. This material may cause the gigahertz peaked radio spectrum in two of the objects, through either free-free absorption or synchrotron self-absorption, due to the increased compactness. Intracluster material may also be linked to the extreme luminosity of these objects through enhancement of the accretion rate due to Compton cooling of the flow by the quasar continuum. Absorption by a Zel'dovich pancake or protocluster is another possibility, which may imply a lower bound on the enrichment of the material and/or limit the geometry of the material.

The quasar with no detected X-ray absorption has intervening damped Ly $\alpha$ absorbers. The X-ray measurement implies a limit on the neutral fraction for these systems, $N(\mathrm{H}$ 1) $/ N(\mathrm{H})>4 \times 10^{-1}(3 \sigma)$, for solar abundances, and a size less than $3 \mathrm{Mpc}$.

The lack of an X-ray redshift prevents us from knowing whether, with X-ray absorption toward high-redshift quasars, we are studying clusters at $z=3$ or disk galaxies (the preferred explanation for damped Ly $\alpha$ systems) at $z \sim 1$. High-resolution spectroscopy around the redshifted oxygen K-edge $(0.2-0.5$ $\mathrm{keV}$ ) is the most promising diagnostic. Also high-resolution (arcsecond) X-ray imaging could separate a quasar from any surrounding cooling flow.

We compared the 1-10 $\mathrm{keV}$ (emitted) spectral indices of the $z=3$ quasars with spectra in the same emitted energy range from low $z$ radio-loud quasars. They show no evidence for changes in X-ray slope greater than 0.3 (99\% confidence), either between $z=0.1$ and $z=3$, or from $L_{X}=10^{44}$ ergs s $^{-1}$ to $L_{X}=10^{48} \mathrm{ergs} \mathrm{s}^{-1}$. This tends to favor short lifetime models for quasars.

Limits of $0.7-1.5 \mathrm{keV}$ were placed on the equivalent width of the Fe-K $6.4 \mathrm{keV}$ fluorescence emission for all four objects.

The large derived X-ray luminosities of two of these quasars (the GPS quasars PKS 0420-388 and PKS 2126-158) are likely to be true $4 \pi$ values, unaffected by relativistic beaming. Such luminosities imply extreme masses $\left(\sim 10^{10-11} M_{\odot}\right)$ for the presumed massive black holes at their centers, and predict strong limits on any X-ray variability ( $\sim 4 \mathrm{yr}$, observed frame).

We thank Sergio Colafrancesco for discussions on cluster formation scenarios. We also thank Mark Birkinshaw, Pepi Fabbiano, Harvey Tananbaum, and the referee, Claude Canizares, for critical readings of the manuscript. This research has made use of the NASA/IPAC Extragalactic Database (NED) which is operated by the Jet Propulsion Laboratory, California Institute of Technology, under contract with the National Aeronautics and Space Administration. This research has also made use of the Einstein On-Line Service, Smithsonian Astrophysical Observatory. This work was supported by NASA grants NAGW-2201 (LTSARP), NAG 5-1872 and NAG 5-1536 (ROSAT), and NASA contracts NAS 5-30934 (RSSDC), NAS 5-30751 (HEAO 2), and NAS 8-39073 (ASC). 
Allen, S. W., \& Fabian, A. C. 1992, MNRAS, 258, 29P

Arnaud, K. A., et al. 1987, MNRAS, 227, 241

Arnaud, M., Hughes, J. P., Forman, W., Jones, C., Lachieze-Rey, M., Yamashita, K., \& Hatsukade, I. 1992, ApJ, 390, 345

Atwood, B., Baldwin, J. A., \& Carswell, R. F. 1985, ApJ, 292, 58

Awaki, H. 1992, Ph.D. thesis, Nagoya Univ.; also ISAS Research Note RN473

Bahcall, J., \& Peebles, J. 1969, ApJ, 156, L7

Bajtlik, S., Duncan, R. C., \& Ostriker, J. P. 1988, ApJ, 327, 570

Bechtold, J., Elvis, M., Fiore, F., Kuhn, O., Cutri, R. M., McDowell, J. C., Rieke, M., Siemiginowska, A., \& Wilkes, B. J. 1994, ApJ, submitted

Boyle, B. J., Fong, R., \& Shanks, T. 1987, MNRAS, 227, 717

Boyle, B. J., Griffiths, R. E., Shanks, T., Stewart, G. C., \& Georgantopoulos, I. 1993, MNRAS, 260, 49

Canizares, C. R., \& Kruper, J. 1984, ApJ, 278, L99

Canizares, C. R., \& White, J. L. 1989, ApJ, 339, 27

Condon, J. J., Condon, M. A., Jauncey, D. L., Smith, M. G., Turtle, A. J., \& Wright, A. E. 1981, ApJ, 244, 5

Davis, M., Efstathiou, G. G., Frenk, C. S., \& White, S. D. M. 1985, ApJ, 292, 371

Ellingson, E., Yee, H. K. C., \& Green, R. F. 1991, ApJS, 76, 455

Ellis, R. S. 1992, in IAU Symp. 149, The Stellar Population of Galaxies, ed. B. Barbuy \& A. Renzini (Dordrecht: Kluwer), 297

Elvis, M., Lockman, F. J., \& Wilkes, B. J. 1989, AJ, 97, 777

Elvis, M., Wilkes, B. J., McDowell, J. C., Green, R. F., Bechtold, J., Willner, S. P., Oey, M. S., Polomski, E., \& Cutri, R. 1994, ApJS, submitted

Fabian, A. C., Hu, E. M., Cowie, L. L., \& Grindlay, J. 1981, ApJ, 248, 47

Fabian, A. C. \& Crawford, C. S. 1990, MNRAS, 247, 439

Fall, M., \& Pei, Y. 1993, ApJ, 402, 479

Field, G. B., \& Perrenod, S. C. 1977, ApJ, 215, 717

Fiore, F., Elvis, M., Mathur, S., Wilkes, B. J., \& McDowell, J. C. 1993, ApJ, 415, 129

Fiore, F., Perola, G. C., Matsuoka, M., Yamauchi, M., \& Piro, L. 1992, A\&A, 262,37

Fireman, E. L. 1974, ApJ, 187, 57

Fugmann, W., \& Meisenheimer, K. 1988, A\&AS, 76, 145

George, I. M., \& Turner, T. J., eds. 1992, NASA GSFC OGIP Calibration Memo

Giallongo, E., Cristiani, S., \& Trevese, D. 1992, ApJ, 398, L9

Gunn, J. E. 1990, in Clusters of Galaxies ed. W. R. Oegerle, M. J. Fitchett, \&

L. Danly (Cambridge: Cambridge Univ. Press), 34

Gunn, J. E., \& Peterson, B. A. 1965, ApJ, 142, 1633

Halpern, J. P. 1984, ApJ, 281, 90

Harris, D. E., et al. 1990, The Einstein Observatory Catalog of IPC X-Ray Sources (CD-ROM: Einstein Data Center, Smithsonian Astrophysical Observatory)

Hasinger, G., \& Snowden, M. 1992, private communication

Heiles, C., \& Cleary, M. N. 1979, Australian J. Phys. Suppl., 47, 1

Hyland, A. R., \& Allen, D. A. 1982, MNRAS, 199, 943

Impey, C., \& Tapia, S. 1990, ApJ, 354, 124

Inda, A., et al. 1993, ApJ, submitted

Jauncey, D. L., Wright, A. E., Peterson, B. A., \& Condon, J. J. 1978, ApJ, 223, L1

Kaastra, J. S., Kunieda, H., \& Awaki, H. 1991, A\&A, 242, 27

Kaiser, N. 1984, ApJ, 284, L49

Kühr, H. Witzel, A., Pauliny-Toth, I. I. K., \& Nauber, U. 1981, A\&AS, 45, 367

Kruper, J. S., Urry, C. M., \& Canizares, C. R. 1990, ApJS, 74, 347

Laor, A., \& Draine, B. T. 1993, ApJ, 402, 441

Lanzetta, K. M., Wolfe, A. M., Turnshek, D. A., Lu, L., McMahon, R. G., \& Hazard, C. 1991, ApJS, 77, 1

Lanzetta, K. M., Turnshek, D. A., \& Wolfe, A. M. 1987, ApJ, 322, 739

Lawrence, A., \& Elvis, M. 1982, ApJ, 256, 410

Lawson, A. J., Turner, M. J. L., Williams, O. R., Stewart, G. C., \& Saxton, R. D. 1992, MNRAS, 259, 743

Lu, L., Wolfe, A. M., \& Turnshek, D. A. 1991, ApJ, 367, 19

Madejski, G. M., Mushotzky, R. F., Weaver, K. A., Arnaud, K. A., \& Urry, C. M. 1991, ApJ, 370, 198

Maoz, D., Bahcall, J. N., Doxsey, R., Schneider, D. P., Bahcall, N. A., Lahav, O., \& Yanny, B. 1992, ApJ, 394, 51
Marscher, A. P. 1988, ApJ, 334, 552

Marshall, F. E., et al. 1993, ApJ, 405, 168

Mather, J. C., et al. 1990, ApJ, 354, L37

Meyer, D. M., \& Roth, K. C. 1990, ApJ, 363, 57

Morrison, R., \& McCammon, D. 1983, ApJ, 270, 119

Morton, D. C., Savage, A., \& Bolton, J. G. 1978, MNRAS, 185, 735

Nandra, K., \& Pounds, K. A. 1992, Nature, 356, 215

Nandra, K., Pounds, K. A., \& Stewart, G. C. 1991, in Iron Line Diagnostics in X-ray Sources, ed. A. Treves, G. C. Perola, \& L. Stella (Berlin: Springer), 177 Nandra, K. 1992, Ph.D. thesis, Univ. of Leicester

O'Dea, C. P. 1990, MNRAS, 245, 20P

O'Dea, C. P., Baum, S. A., \& Stanghellini, C. 1991, ApJ, 380, 66

Osmer, P. S., \& Smith, M. G. 1977, ApJ, 215, L47

Pan, H. C., Stewart, G. C., \& Pounds, K. A. 1990, MNRAS, 242, 177

Pearson, T. J., \& Readhead, A. C. S. 1984, in IAU Symp. 110, VLBI and Compact Radio Sources, ed. R. Fanti, K. Kellerman, \& G. Setti (Dordrecht: Reidel), 15

Peebles, P. J. E. 1993, Principles of Physical Cosmology (Princeton: Princeton Univ. Press), 550

Pettini, M., Boksenberg, A., \& Hunstead, R. W. 1990, ApJ, 348, 48

Pei, Y., Fall, M., \& Bechtold, J. 1991, ApJ, 378, 6

Peterson, B. A., Savage, A., Jauncey, D. L., \& Wright, A. E. 1982, ApJ, 260, L27

Pfefferman, E., et al. 1987, Proc. SPIE, 733, 519

Phillips, R. B., \& Mutel, R. L. 1982, A\&A, 106, 2

Pounds, K. A. 1990, MNRAS, 242, 20P

Raymond, J., \& Smith, B. W. 1977, ApJS, 35, 419

ROSAT Mission Description. 1991, NASA NRA 91-OSSA-3, Appendix F

ROSAT Newsletter, 1992, No. 10

Rybicki, G. B., \& Lightman, A. P. 1979, Radiative Processes in Astrophysics (New York: Wiley)

Sargent, W. L. W. 1988, in QSO Absorption Lines, ed. J. C. Blades, D. Turnshek, \& C. A. Norman (Cambridge: Cambridge Univ. Press), 1

Sargent, W. L. W., Steidel, C., \& Boksenberg, A. 1990, ApJ, 391, 364

Schlegel, E. 1993, private communication

Schneider, D. P., et al. 1992, PASP, 104, 678

Schneider, D. P., Schmidt, M., \& Gunn, J. E. 1989, AJ, 98, 1507

Schreier, E. J., Burns, J. O., Gorenstein, P., \& Feigelson, E. D. 1982, ApJ, 261, 42

Shapiro, P. R., \& Bahcall, J. N. 1980, ApJ, 241, 1

Shastri, P., Wilkes, B. J., Elvis, M., \& McDowell, J. 1993, ApJ, 410, 29

Singh, K. P., Rao, A. R., \& Vahia, M. N. 1990, MNRAS, 246, 706

Stark, A. A., Gammie, C. F., Wilson, R. W., Bally, J., Linke, R., Heiles, C., \& Hurwitz, M. 1989, ApJS, 79, 77

Subramanian, K., \& Swarup, G. 1992, Nature, 359, 512

Trümper, J. 1983, Adv. Space Res., 2, No. 4, 241

Turner, T. J., Done, C., Mushotzky, R., Madejski, G., \& Kunieda, H. 1992, ApJ, 391, 102

Turner, T. J., \& Pounds, K. A. 1989, MNRAS, 240, 833

Turnshek, D. A., Wolfe, A. M., Lanzetta, K. M., Briggs, F. H., Cohen, R. D., Foltz, C. B., Smith, H. E., \& Wilkes, B. J. 1989, ApJ, 344, 567

Uson, J. M., Bagri, D. S., \& Cornwell, T. J. 1991, Phys. Rev. Lett., 67, 3328

Véron-Cetty, M.-P., \& Véron, P. 1991, ESO Sci. Rep. no. 10

Wagoner, R. 1967, ApJ, 149, 465

White, D. A., Fabian, A. C., Johnstone, R. M., Mushotzky, R. F., \& Arnaud, K. A. 1991, MNRAS, 252, 72

Wilkes, B. J., \& Elvis, M. 1987, ApJ, 323, 243

Wilkes, B. J., Elvis, M., Fiore, F., McDowell, J. C., Tananbaum, H., \& Lawrence, A. 1992, ApJ, 393, L1

Wilkes, B. J., Tananbaum, H., Worrall, D. M., Avni, Y., Oey, M. S., \& Flanagan, J. 1994, ApJS, in press

Wills, B. J., Wills, D., Breger, M., Antonucci, R. R. J., \& Barvainis, R. 1992, ApJ, 398, 454

Williams, O. R., et al. 1992, ApJ, 389, 157

Wolfe, A. M. 1988, in QSO Absorption Lines, ed. J. C. Blades, D. Turnshek, \& C. A. Norman (Cambridge: Cambridge Univ. Press), 297

Worrall, D. M., \& Wilkes, B. J. 1990, ApJ, 360, 396

Yee, H. K. C., \& Green, R. F. 1984, ApJ, 280, 79 